\title{
Surfactant Assisted Rapid-Release Liposomal Strategies Enhance the Antitumor Efficiency of Bufalin Derivative and Reduce Cardiotoxicity
}

\author{
Lina Gao' \\ Lei Zhang' \\ Fengjun $\mathrm{He}^{\prime}$ \\ Jing Chen' \\ Meng Zhao' \\ Simin $\mathrm{Li}^{\prime}$ \\ Hao Wu (ID ' \\ Yumeng Liu' \\ Yinan Zhang ${ }^{1,2}$ \\ Qineng Ping ${ }^{3}$ \\ Lihong $\mathrm{Hu}^{1,2}$ \\ Hongzhi Qiao (D) 1,2,4 \\ 'Jiangsu Key Laboratory for Functional \\ Substance of Chinese Medicine, Nanjing \\ University of Chinese Medicine, Nanjing, \\ 210023, People's Republic of China; \\ ${ }^{2}$ State Key Laboratory Cultivation Base \\ for TCM Quality and Efficacy, School of \\ Pharmacy, Nanjing University of Chinese \\ Medicine, Nanjing, 210023, People's \\ Republic of China; ${ }^{3}$ Department of \\ Pharmaceutics, State Key Laboratory of \\ Natural Medicines, China Pharmaceutical \\ University, Nanjing, 210009, People's \\ Republic of China; ${ }^{4}$ Jiangsu Engineering \\ Research Center for Efficient Delivery \\ System of TCM, Nanjing University of \\ Chinese Medicine, Nanjing, 210023, \\ People's Republic of China
}

Correspondence: Hongzhi Qiao; Lihong $\mathrm{Hu}$

State Key Laboratory Cultivation Base for TCM Quality and Efficacy, School of Pharmacy, Nanjing University of Chinese Medicine, I 38 Xianlin Avenue, Nanjing, 210023, People's Republic of China Email qiaohz@njucm.edu.cn; Ihhu@njucm.edu.cn
Background: BF211, a derivative of bufalin (BF), shows significantly improved solubility and potent antitumor efficiency compared to BF. Unfortunately, the unwanted toxicity such as cardiotoxicity caused by unspecific distribution has hindered its clinical use.

Methods: PEGylated BF211 liposomes (BF211@Lipo) were designed and optimizely prepared based on the pre-prescription research. In vitro and in vivo cardiotoxicity was evaluated. In vivo pharmacokinetics and biodistribution of BF211@Lipo were investigated. In vivo antitumor activity and toxicity were evaluated in HepG2 cell xenograft models. The rapid-release triggered by Poloxamer 188 (P188) was assessed in vitro and in vivo.

Results: The optimized BF211@Lipo displayed a spherical morphology with a size of $(164.6 \pm 10.3) \mathrm{nm}$ and a high encapsulation efficiency of $(93.24 \pm 2.15) \%$. The in vivo concentration-time curves of BF211 loaded in liposomes showed a prolonged half-life in plasma and increased tumor accumulation. No obvious abnormality in electrocardiograms was observed in guinea pigs even at $9 \mathrm{mg} / \mathrm{kg}$. Moreover, to improve the efficient release of BF211@Lipo, a surfactant-assisted rapid-release strategy was developed, and the releasepromoting mechanism was revealed by the fluorescence resonance energy transfer (FRET) and fluorescence nanoparticle tracking analysis (fl-NTA) technology. Sequential injection of BF211@Lipo and P188 could ignite the "cold" liposomes locally in tumor regions, facilitating the burst release of BF211 and enhancing the therapeutic index.

Conclusion: Our progressive efforts that begin with preparation technology and dosage regimen enable BF211 to like a drug, providing a promising nano platform to deliver the cardiac glycosides and alleviate the side effects by decreasing unspecific biodistribution.

Keywords: bufalin derivative, liposome, rapid-release, antitumor, cardiotoxicity

\section{Introduction}

Bufalin (BF) is one of the key active components of the traditional Chinese medicine $\mathrm{ChanSu}$, which is derived from secretions from the skin and parotid venom glands of Bufo gargarizans Cantor or Bufo melanostictus Schneider. ${ }^{1}$ As a typical representative of cardiac glycosides, BF recently has been found to have substantial anticancer activity. ${ }^{2}$ However, the poor solubility and severe side effects hampered its extensive clinical use. ${ }^{3}$ BF211 is a derivative of BF, which has previously been synthesized and commonly used as hydrochloride. BF211 exhibited significantly improved solubility (increased from $10 \mu \mathrm{g} / \mathrm{mL}$ to $2500 \mu \mathrm{g} / \mathrm{mL}$ ) and stronger cytotoxicity against cancer cells than $\mathrm{BF}$, eg, the half maximal inhibitory concentration $\left(\mathrm{IC}_{50}\right)$ of $\mathrm{BF} 211$ against the human liver hepatocellular carcinoma cell-line HepG2 was 18-fold lower than that 
of $\mathrm{BF}^{4}$ The underlying anticancer mechanisms mainly involved proliferation inhibition through the activation of signaling cascades downstream of the $\mathrm{Na}^{+} / \mathrm{K}^{+}$-ATPase signalosome, ${ }^{5}$ and apoptosis induction through the downregulation of cyclin A, Bcl-2 and Bcl-xL. ${ }^{6,7}$ Unfortunately, its side effects, particularly cardiotoxicity, have not yet been subdued (Figure 1A).

Liposomes are considered an effective method to improve the therapeutic efficacy and reduce the toxicity owing to their biocompatibility, prolonged blood circulation time and target-oriented delivery of precise medicines. ${ }^{8}$ The clinical performance of Doxil ${ }^{\circledR}$, which was the first FDAapproved liposomal doxorubicin (Dox) in 1995, against various malignant tumors is superior to that of free Dox accompanied by the improved overall patient compliance. ${ }^{9}$ Of all the submissions to the FDA for nanodrug products (1973-2015), liposomes were the most prevalent category (33\% of drug products), demonstrating enduring attraction and development value. ${ }^{10}$ Hence, liposome formulation of BF211 was proposed to diminish its cardiotoxicity and enhance its therapeutic effect.

To realize the clinical transformation of liposomal drugs, sufficient and stable drug loading into liposomes is an obligatory requirement. In most cases, owing to the extremely limited space of liposomes, remote (active) loading of drugs may be the only approach to achieve a high drug concentration. ${ }^{11}$ Remote loading uses an ion gradient as the driving force for guiding drugs into
A

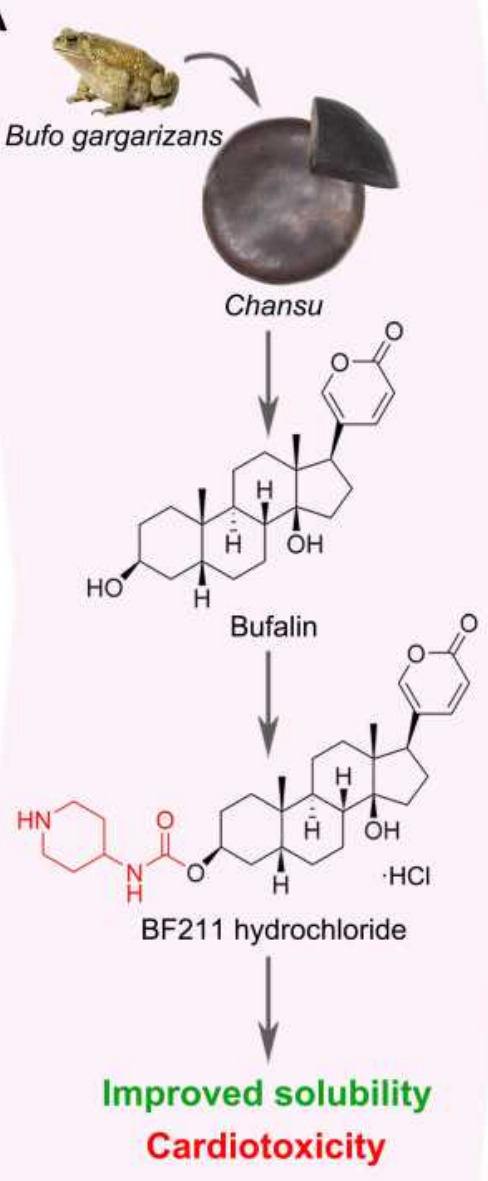

B

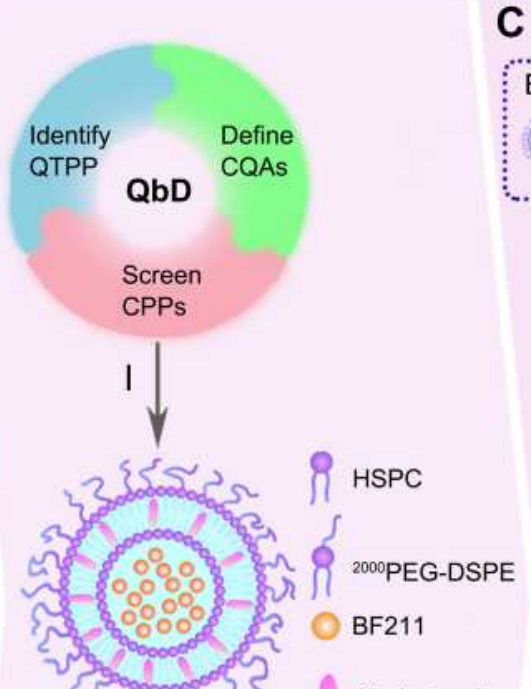

Cholesterol

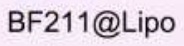

II
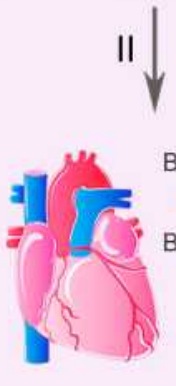

$\mathrm{BF} 211(\mathrm{aq})$ BF211@Lipo

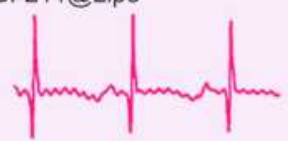

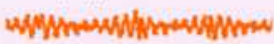

C
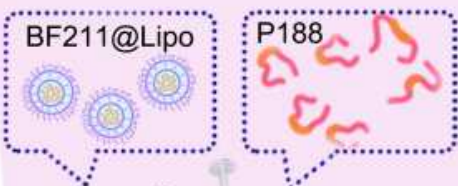

III

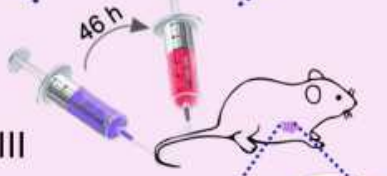

42

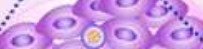

ionos.

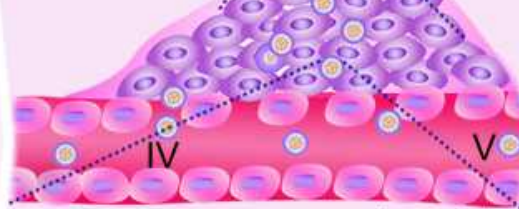

Extracellar matrix
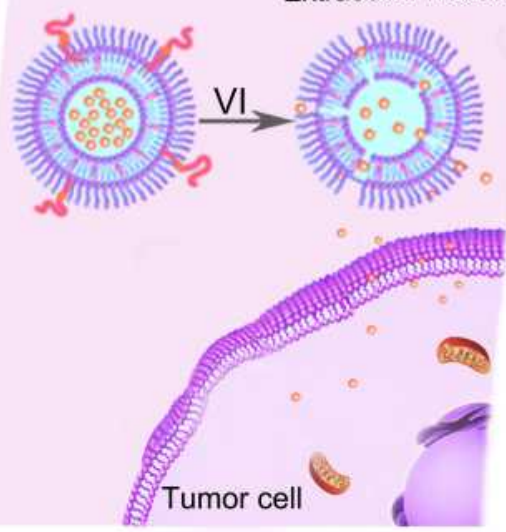

\section{I.Stealth liposomes preparation based on QbD II.Reduced cardiotoxicity III.Sequential}

\section{administration IV.EPR effect V.Long blood circulation VI.P188 triggered rapid-release}

Figure I Schematic representation of liposomal BF2II and surfactant-assisted rapid release technologies for enhanced antitumor efficacy and reduced cardiotoxicity. (A) The evolution of bufalin derivatives from natural active ingredients through chemical modification. BF2II manifests the improved solubility and the remaining cardiotoxicity. (B) Stealth liposomal BF2II (BF2I I@Lipo) fabricated based on the Quality by Design (QbD) strategies for the prolonged blood circulation time and reduced cardiotoxicity. (C) Sequential injection of BF2II@Lipo and PI88 achieves surfactant-assisted rapid-release of liposomes, further boosting the anti-tumor efficiency. 
prefabricated liposomes, and it is suitable for amphipathic weak acids or bases. ${ }^{12}$ BF211 is an amphipathic weak base that can be ionized and charged or nonionized and uncharged at a certain $\mathrm{pH}$, accompanied by a change in water solubility ${ }^{4}$ (Figure 1). Drug loading can be achieved through a base exchange of the weak base BF211 with a transmembrane ion gradient, eg, ammonium ions. ${ }^{13}$

Another challenge for liposomes is the insufficient effective drug concentration at tumor sites and the unsatisfactory clinical efficacy due to deficient drug release from liposomes. ${ }^{14}$ Although the systemic injection of liposomes results in increased tumor accumulation through the enhanced permeability and retention (EPR) effects, a considerable proportion of drug-containing liposomes remain inactive in the tumor interstitial space. Intact liposomes are reported to move only 3 to 5 cells away from vascular regions $;{ }^{15}$ therefore, the cellular uptake and penetration of intact liposomes is restricted until the drug is released in the free form. ${ }^{16}$ Accumulating evidence indicates that surfactants can affect the integrity and stability of lipid bilayers in different ways. Conventional anionic surfactants can make bilayers collapse and remove them from the surface, while nonionic surfactants are able to insert into and gradually replace lipid bilayers. The perturbance of surfactants into lipid bilayers often increases the permeability of these membranes, leading to the accelerated release of liposomal compounds. ${ }^{17}$

In this paper, for the first time, the developmental details of liposomal BF211 were disclosed, including formulation and process optimization, improvement of cardiac toxicity, pharmacokinetics (PK) and tissue distribution results in animal models, and in particular the advantages of antitumor pharmacodynamics over the BF211 solution and the commercially available Dox liposomes. In addition, the assembly behavior and morphology characteristics of BF211 in different solution environments were presented, and the existence form of the drug in liposomes was studied. Furthermore, in view of the problem of insufficient release of liposomes, a surfactant-assisted liposome release technology was developed, and the release-promoting mechanism was studied by FRET technology, demonstrating the correlation between release promotion and activity improvement. The outcome of this study highlights the potential of liposomal BF211 for antitumor treatment and supports the further clinical development of this effective therapeutic approach.

\section{Materials and Methods}

\section{Materials}

BF211 was synthesized in our laboratory as previously described. $^{4}$ Distearoyl phosphoethanolamine-PEG ${ }^{2000}$ $\left({ }^{2000}\right.$ PEG-DSPE), cholesterol (CH), hydrogenated soybean phospholipids (HSPC) were obtained from Lipoid GmbH (Ludwigshafen, Germany). Ammonium sulfate was purchased from Nanjing Chemical Reagent Company (Nanjing, China). 3-(4, 5-dimethylthiazol-2-yl)-2, 5-diphenyltetrazolium bromide (MTT), 4\% paraformaldehyde (PFA), 4',6-diamidino-2-phenylindole (DAPI), DiD, DiR, DiI, PKH-26, trypsin-EDTA solution, DMEM and RPMI 1640 were supplied by KeyGEN BioTECH Co. Ltd (Nanjing, China). Creatine Kinase (CK) kit and lactate dehydrogenase (LDH) kit, Doxorubicin (Dox) were purchased from Nanjing JinYibai Biological Technology Co. Ltd. (Nanjing, China). Cinobufagin was supplied by Sigma Corporation (St. Louis, MO, USA). Poloxamer 188 (P188) (PEO/PPO/PEO ratio is 80/27/80, MW 8600) and Tween 80 for injection were purchased from Feiyu BioTECH Co. Ltd (Nanjing, China). HPLC-grade methanol (MeOH), acetonitrile $(\mathrm{ACN})$ and formic acid were obtained from Merck (Darmstadt, Germany). Deionized (DI) water produced by a Milli-Q ${ }^{\circledR}$ Plus System (Billerica, MA, USA) with a resistivity of $18.2 \mathrm{~m} \Omega \cdot \mathrm{cm}-1$ was used in all the experiments. All other chemicals and reagents were obtained from Sigma Aldrich (Shanghai, China), and used without further purification.

\section{Cells and Animals}

Human liver hepatocellular carcinoma (HepG2) cells, Raw264.7 macrophage cell, rat myocardial cells (H9C2) were obtained from American Type Culture Collection (ATCC), cultured at $37^{\circ} \mathrm{C}$ under a humidified atmosphere of 5\% CO2 in DMEM or 1640 medium supplemented with $10 \%$ fetal bovine serum (FBS), $100 \mathrm{IU} / \mathrm{mL}$ penicillin, and $100 \mu \mathrm{g} / \mathrm{mL}$ streptomycin.

BALB/c mice and BALB/c nude mice (18-22 g), pigmented guinea pig (220-250 g), and Sprague-Dawley (SD) rats (220-250 g) were purchased from Nanjing Qinglongshan Animal Breeding Company (Nanjing, China). The animals were housed with ad libitum access to food and water at $25^{\circ} \mathrm{C}$ and relative humidity of $55 \%$. Animal study protocol was approved by Animal Ethics Committee of Nanjing University of Chinese Medicine, and performed in accordance to Guide for Care and Use of Laboratory Animals. 


\section{Preparation of BF2 I I-Loaded Liposomes (BF2I I@Lipo)}

The liposomes were prepared by the thin-film dispersion method with the ammonium sulfate gradient technique to actively load the drugs. ${ }^{18}$ In brief, HSPC, ${ }^{2000}$ PEG-DSPE and $\mathrm{CH}$ were dissolved in ethanol completely, rotary evaporated at $50^{\circ} \mathrm{C}$ to form a thin film, dried overnight under vacuum and hydrated with $0.11 \mathrm{M}$ ammonium sulfate solution. Size reduction and homogenization of liposomes were performed by ultrasonic treatment using ultrasonic probes. The outside ammonium sulfate was replaced by DI water by gel exclusion chromatography with a Sephadex G50 column, and the blank liposome (Lipo) was incubated with $2 \mathrm{mg} / \mathrm{mL} \mathrm{BF} 211$ (aq) overnight with stirring at $50^{\circ} \mathrm{C}$. BF211@Lipo was obtained after removing the unencapsulated drug by Sephadex G50 column. Formulations and processes were systematically optimized in accordance with the scientific approach of QbD. ${ }^{19,20}$ The detailed parameters and process are shown in the supporting information.

\section{Characterization of BF2II@Lipo}

The particle size, polydispersity index (PDI) and zeta potential of BF211@Lipo were measured with a Zetasizer (Malvern Instruments, Worcestershire, UK). The morphology of BF211@Lipo was characterized with an HT7700 transmission electron microscope (TEM) (HITACHI, Tokyo, Japan). The concentration of BF211 was measured by an HPLC assay (Waters 2695 System, Milford, MA, USA). Specific conditions were as follows: Hedera ${ }^{\circledR}$ ODS- 2 column $(4.6 \mathrm{~mm} \times 250 \mathrm{~mm}, 5 \mu \mathrm{m})$; mobile phase, methanol $/ 0.3 \%$ phosphoric acid in water $(60: 40, \mathrm{v} /$ v); flow rate, $1.0 \mathrm{~mL} / \mathrm{min}$; column temperature, $30^{\circ} \mathrm{C}$; detection wavelength, $300 \mathrm{~nm}$; and injection volume, 10 $\mu \mathrm{L}$. The encapsulation efficiency and drug loading were calculated by the following formulas.

$$
\begin{aligned}
& \text { Encapsulation efficiency }(E E, \%) \\
& =\frac{\text { Weight of BF } 211 \text { in liposomes }}{\text { Initial weight of BF } 211} \times 100 \%
\end{aligned}
$$

$$
\begin{aligned}
\operatorname{Drug} \text { loading }(D L, \%)= & \frac{\text { Weight of BF211 in liposomes }}{\text { Total weight of BF211@Lipo }} \\
& \times 100 \%
\end{aligned}
$$

The storage stability of BF211@Lipo was evaluated by monitoring the particle size and drug leakage at $4{ }^{\circ} \mathrm{C}$ for one month. Liposomal stability in complex biological samples (serum or tumor homogenates) was determined by incubation with $75 \%$ FBS or homogenates $(1: 1, v / v)$ at $37^{\circ} \mathrm{C}$ for $48 \mathrm{~h}$ using fl-NTA technology (NS300, Malvern, United Kingdom).

Liposomal BF211, BF211(aq) or a physical mixture of BF211 and empty liposomes were placed in a $1 \mathrm{~mm}$ cuvette for circular dichroism (CD). The CD spectra were obtained at room temperature from $260 \mathrm{~nm}$ to $150 \mathrm{~nm}$ at a scan speed of $50 \mathrm{~nm} / \mathrm{min}$ on a J-810 spectropolarimeter (Jasco, Easton, MD), and five scans were accumulated and averaged. Differential scanning calorimetry (DSC) was carried out using a Netzsch 204 instrument (NetzschGeraetebau GmbH, Selb, Germany). Samples were heated from room temperature to $300^{\circ} \mathrm{C}$ at a heating rate of $10^{\circ} \mathrm{C} /$ min. Thermal events were determined from the obtained thermograms using Netzsch software.

\section{Release Profile of BF2II@Lipo}

Drug release from BF211@Lipo was carried out in PBS media (pH 5.0, 6.5 and 7.4) using the dialysis method. BF211@Lipo (2.0 mg BF211) was placed into a dialysis bag (molecular weight cutoff: $3500 \mathrm{Da}$ ), immersed in $50 \mathrm{~mL}$ PBS medium, and gently agitated in a tabletop air bath incubator at $37^{\circ} \mathrm{C}$. At predetermined intervals, a dissolution sample of $5 \mathrm{~mL}$ was collected, followed by replenishment with fresh PBS medium. BF211 $(2.0 \mathrm{mg})$ dissolved in DI water also served as a control for drug release. The concentration of BF211 in dissolution samples was quantified by HPLC.

\section{Cardiotoxicity and Acute Toxicity Assessment}

To evaluate the toxicity-reducing effect of BF211@Lipo, a series of safety tests were carried out. Electrocardiogram (ECG) assays were performed on pigmented guinea pigs to evaluate the cardiotoxicity of BF211@Lipo. In brief, 27 male guinea pigs were divided into 3 groups, the BF211@Lipo and BF211(aq) groups were treated with different doses of 3, 4, 6 and $9 \mathrm{mg} / \mathrm{kg}$, with saline as a control. After $1 \mathrm{~h}$, the animals were anaesthetized with $2 \%(\mathrm{w} / \mathrm{v})$ pentobarbital sodium $(0.25 \mathrm{~mL} / 100 \mathrm{~g})$ via intraperitoneal injection and placed in a supine position. Platinum electrodes of thin microneedles were positioned subcutaneously in the right forelimb (negative pole) and left hind limb (positive pole), and the black electrode was implanted in the right hind limb of grounded guinea pigs. These needles were connected via coaxial cables to the input of two differential Isodam amplifiers (Cardiosta-T, 
Siemens). The signal was amplified, filtered and continuously monitored and recorded at predetermined time intervals. The amplitude and duration of ECG waves were manually measured from digitalized recordings with AD Instruments LabChart flexible data acquisition and analysis software (PowerLab Version: 7.0). The body temperature of the animals was maintained with a $37^{\circ} \mathrm{C}$ heating pad throughout the examination process.

To calculate the lethal dose $50\left(\mathrm{LD}_{50}\right)$ value of the BF211@Lipo, a single-dose acute toxicity study of BF211@Lipo or BF211(aq) was performed in both male and female BALB/c mice. The animals were treated intravenously with a single dose $(10,20,30,35,40,45$, or $60 \mathrm{mg} / \mathrm{kg}$ ) and observed after dosing individually for 14 days. At the end of the experiment, the numbers of dead animals were recorded. The $\mathrm{LD}_{50}$ value was calculated according to the Bliss method. ${ }^{21}$

Cardiac markers and pathological sections were also assessed. In brief, male BALB/c mice were divided into 3 groups, treated intravenously with BF211@Lipo or BF211 (aq) $(7 \mathrm{mg} / \mathrm{kg})$ or saline as a control every other day and monitored for 2 weeks. The mice were sacrificed on day 15 , and blood was withdrawn for CK and $\mathrm{LDH}$ analysis. To further investigate the potency of the liposomes inducing cell death, dual acridine orange/ethidium bromide $(\mathrm{AO} / \mathrm{EB})$ staining was performed in rat healthy myocardial cells (H9C2). After $24 \mathrm{~h}$ treatment of BF211(aq) and BF211@Lipo, the cells were collected and dyed with $\mathrm{AO} / \mathrm{EB}$ reagent according to the manufacturer's instructions. All samples were conducted in triplicate.

\section{In vivo $\mathrm{PK}$ and Tissue Biodistribution of BF2II@Lipo}

The PK of BF211@Lipo and BF211(aq)were investigated in male SD rats. In brief, BF211@Lipo or BF211(aq) was administered intravenously via tail vein injection at $5 \mathrm{mg} /$ $\mathrm{kg}$, and $0.3 \mathrm{~mL}$ blood was collected from the orbital cavity at predetermined time intervals. The samples were centrifuged at $6000 \mathrm{rpm}$ for $10 \mathrm{~min}$ at $4^{\circ} \mathrm{C}$ to obtain plasma. Cinobufagin, used as the internal standard for calibration and quality control, was added to the plasma sample and vortexed for $30 \mathrm{~s}$. Then, $400 \mu \mathrm{L}$ protein precipitant solvent ( $\mathrm{MeOH} / \mathrm{ACN} ; 1: 1, \mathrm{v} / \mathrm{v}$, containing $0.6 \%$ formic acid) was added to the sample to precipitate the proteins and extract the analytes. After centrifugation, the supernatant was concentrated and redissolved in $100 \mu \mathrm{L} \mathrm{MeOH}$ for analysis with UPLC Xevo TQD (Waters Corp., Milford, MA,
US A). A quality control (QC) sample was prepared and determined discontinuously during the analysis. Specific UPLC conditions were as follows: ACQUITY ${ }^{\mathrm{TM}} 1.7 \mu \mathrm{m}$ BEH C18 column (Waters Corp., Milford, MA, USA); mobile phase, (A) DI water with $0.3 \%(\mathrm{v} / \mathrm{v})$ formic acid and (B) $\mathrm{MeOH}$. The detailed gradient conditions were as follows: 0-5 $\mathrm{min}, 90-10 \% \mathrm{~A}$; 5-6 $\mathrm{min}, 10-90 \% \mathrm{~A} ; 6-6.5$ min, $90 \% \mathrm{~A}$; flow rate, $0.3 \mathrm{~mL} / \mathrm{min}$; column temperature, $40^{\circ} \mathrm{C}$; and injection volume, $10 \mu \mathrm{L}$. The mass spectrometry conditions were as follows: ion mode, positive; desolvation gas (nitrogen), $800 \mathrm{~L} / \mathrm{h}$ at $300^{\circ} \mathrm{C}$; cone gas (nitrogen), $50 \mathrm{~L} / \mathrm{h}$; source temperature, $100^{\circ} \mathrm{C}$; capillary voltage, $3.85 \mathrm{kV}$; cone voltage, $35 \mathrm{~V}$. Data between m/z 50 and 600 were recorded in centroid mode.

For biodistribution studies, HepG2 xenograft mice were established. Briefly, $5 \times 10^{6}$ HepG 2 cells were injected subcutaneously into the right forelimb armpit of BALB/c nude mice. Tumor volume $(\mathrm{V})$ was calculated using $\mathrm{V}=\mathrm{L} * \mathrm{~W}^{2} / 2$, where $\mathrm{L}$ is the length of the tumor and $\mathrm{W}$ is the width of the tumor. When the tumor size reached $200-250 \mathrm{~mm}^{3}$, the mice were intravenously injected with DiR-labelled liposomes (DiR-Lipo) or free DiR. ${ }^{22}$ At predetermined time intervals $(1,2,4,8,24$ and $48 \mathrm{~h})$, the mice were anaesthetized with isoflurane and photographed. At $24 \mathrm{~h}$ and $48 \mathrm{~h}$ postinjection, the mice were sacrificed, and the main organs and tumors were collected for imaging.

\section{Antitumor Efficacy of BF2II@Lipo In vitro Cytotoxicity of BF2I I@Lipo}

For MTT analysis, HepG2 cells were treated with Lipo, BF211(aq) or BF211@Lipo at various concentrations. In brief, $2 \times 10^{4}$ cells per well were seeded in a 96-well plate and incubated overnight. When the cells reached $80 \%$ confluence, the medium was replaced with BF211(aq) or BF211@Lipo ranging from 1 to $25 \mathrm{nM}$ and incubated for $24 \mathrm{~h}$. After incubation, MTT was added, and samples were further incubated for $4 \mathrm{~h}$. The insoluble crystal that formed was solubilized using DMSO, and the absorbance was read at $490 \mathrm{~nm}$ using a microplate reader (Tecan Infinite 200 PRO, Austria).

\section{In vivo Antitumor Efficacy of BF2II@Lipo}

The antitumor efficacy of BF211@Lipo was evaluated in HepG2 murine xenograft model $(n=5)$ through a HepG2 xenograft model. ${ }^{23}$ When the tumor volume reached approximately $100 \mathrm{~mm}^{3}$, mice were treated with saline, BF211(aq) (2 mg/kg), BF211@Lipo (2, 4, and 8 mg/kg) or liposomal Dox (Dox@Lipo) (2 mg/kg), as a reference 
product, every other day and monitored for 3 weeks. Animal body weight and tumor volume were recorded. On day 22, animals from each group were euthanized, and the tumors were harvested, washed with saline three times, photographed and fixed in 4\% PFA for HE staining and TUNEL apoptosis staining. The main organs (heart, liver, spleen, lung and kidney) were collected for HE staining.

\section{Surfactant-Assisted Rapid-Release Strategy of Liposomes}

To increase the release efficiency of liposome, a surfactant-assisted rapid release strategy was developed. Two commonly used injectable surfactants, Tween 80 and P188, were used to investigate their effects on the release of liposomes. ${ }^{24}$ The effective concentrations of surfactants triggering liposome release were screened and optimized. In brief, a series of surfactants was mixed with BF211@Lipo and incubated for $2 \mathrm{~h}$ at $37^{\circ} \mathrm{C}$. Then, the released drug was collected by ultrafiltration and determined by HPLC. Additionally, the dialysis method was employed to verify the effect of P188 $(0.05 \%$, w/v) on BF211@Lipo release with tumor homogenates in PBS medium pH 6.5 to simulate the tumor microenvironment.

The interaction mechanism of P188 and liposomes was investigated. P188 $(0.05 \%$, w/v) was mixed with liposomes (or fluorescence-labeled liposomes) and incubated for $2 \mathrm{~h}$ at $37^{\circ} \mathrm{C}$. The size distribution was measured by DLS and fl-NTA. The morphology and structural details were characterized by TEM. To further confirm the interaction, the FRET technique was utilized.

\section{Optimization of Dosage Regimen}

To realize the rapid release of liposomes at the tumor site, the dosage regimen was further optimized. The time at which BF211@Lipo and P188 reached the tumor was monitored using a noninvasive near-infrared imaging system. DiR-Lipo or DiR-labeled P188 (DiR-P188) was prepared and intravenously injected into HepG2 tumorbearing mice, respectively. At predetermined intervals, the mice were anaesthetized with isoflurane and photographed. At $8 \mathrm{~h}, 24 \mathrm{~h}$ and $48 \mathrm{~h}$ post administration, the mice were sacrificed, and the tumors were collected for imaging. Regions of interest (ROIs) were circled around the tissues, and the fluorescence intensity was analyzed using Living Image Software 4.5.2. To further verify the results, UPLC/MS was employed to quantify the BF211 concentration in the tumor tissues.

\section{Antitumor Efficacy of Intermittent Treatment Schedules with BF2II@Lipo and PI88}

The cytotoxicity was evaluated by exposing HepG2 cells to Lipo, P188, BF211@Lipo or BF211@Lipo with P188 for $24 \mathrm{~h}$ followed by MTT assay. To evaluate the in vivo combination therapy of BF211@Lipo and P188 with sequential injection, HepG2 tumor-bearing mice were intravenously treated with P188 $(0.25 \%$, w/v, $200 \mu \mathrm{L} /$ mouse) 46 h post injection of BF211@Lipo at a dose of $2 \mathrm{mg} / \mathrm{kg}$ every five days compared with saline, Lipo, P188, BF211(aq) or BF211@Lipo alone. The mouse body weight and tumor volume were recorded every three days. Finally, the animals were sacrificed, and samples were collected for HE staining.

\section{Statistical Analysis}

All data are presented as the mean \pm standard deviation (SD). One-way or two-way ANOVA was used for the statistical analysis, depending on the study parameters. Statistical significance was indicated as $* p<0.05,{ }^{*} p<$ 0.01 , and $* * * p<0.001$.

\section{Results}

\section{Optimization of BF2I I@Lipo by QbD Approach}

The liposomes were prepared by the thin-film dispersion method with the ammonium sulfate gradient technique (Figure 2A). To optimize the liposomal formulations, we adopted the $\mathrm{QbD}$ approach to optimize the factors that affect the quality of the formulation. ${ }^{25} \mathrm{~A}$ combinatorial library containing different compositions of formulations and process parameters was built, which comprised the definition of the quality target product profile (QTPP) to determine the final liposomal product (Table S1), critical quality attributes (CQAs) to ensure the desired product quality (Table S2) and critical process parameters (CPPs) to monitor or control the preparation process (Table S3). The types of lipids and solvents were determined according to similar commercially available varieties to ensure the safety of production and use. ${ }^{26}$ The range of BF211@Lipo formulations and process parameters were optimized critically, resulting in a final composition of $\mathrm{HSPC} /$ $\mathrm{CH} /{ }^{2000}$ PEG-DSPE (14:5:6, wt $\%$ ). The incorporation ratio of ${ }^{2000}$ PEG-DSPE was determined by a PK study. As shown in Table S4, when the ratio of ${ }^{2000}$ PEG-DSPE to total lipids 


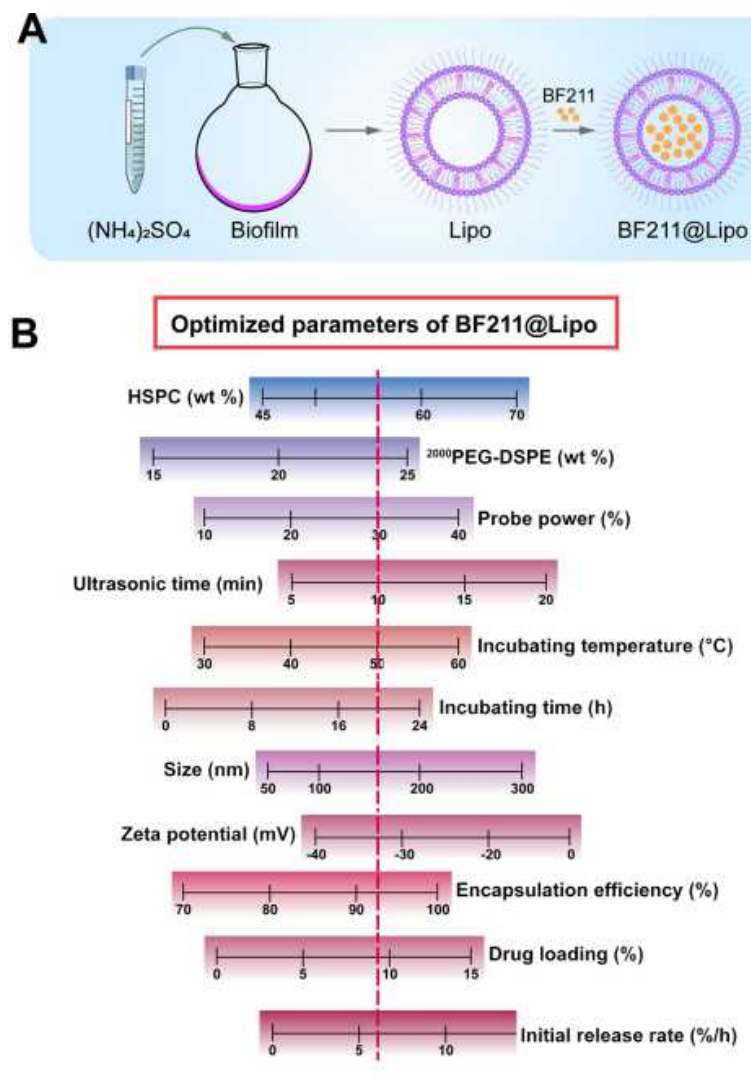

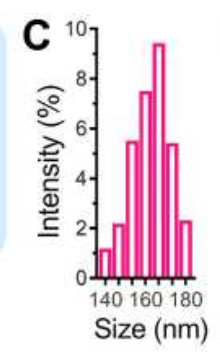
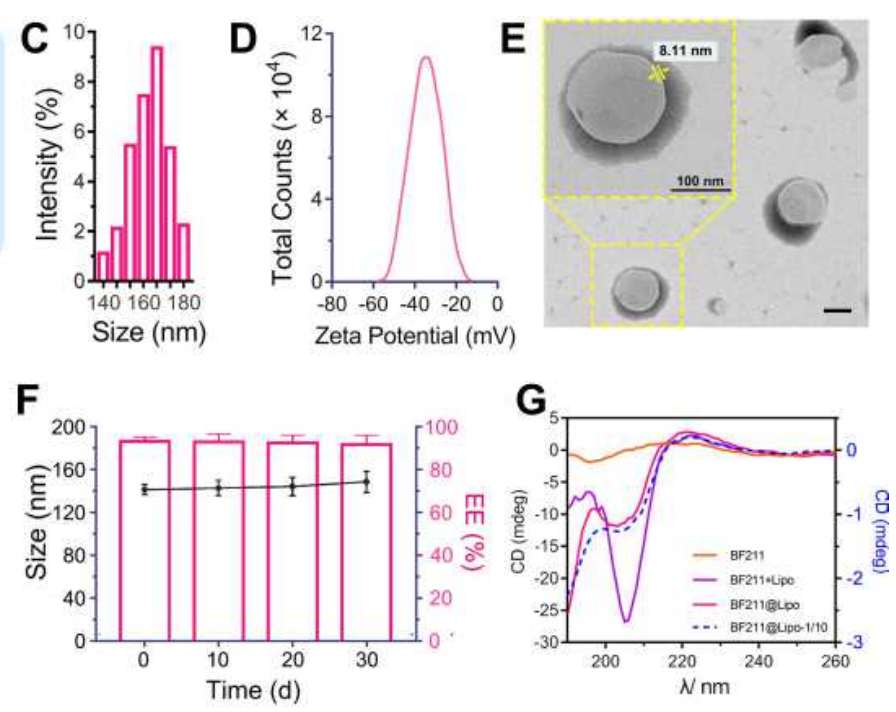

H
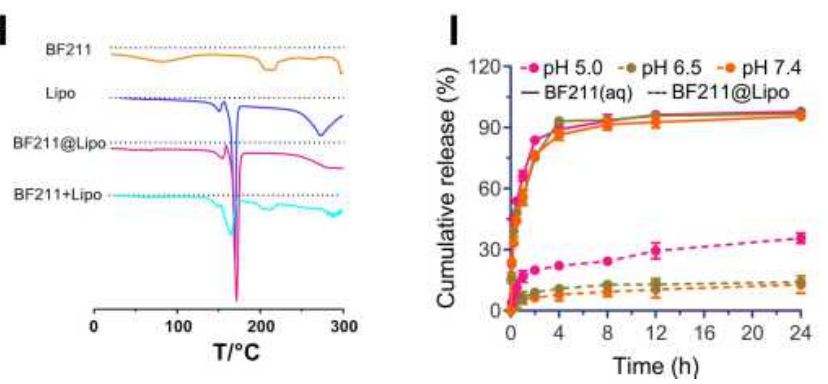

Figure 2 Optimization, characterization and drug release of BF2II@Lipo. (A) Schematic of the BF2II@Lipo prepared by ammonium sulfate gradient method to load BF2II into the blank liposomes (Lipo). (B) Range of formulation parameters and physicochemical properties evaluated during the development of BF2II@Lipo, with optimized BF2II@Lipo parameters indicated by the red dotted line. Particle size distribution (C) and Zeta potential (D) of the optimized BF2II@Lipo by DLS. (E) Morphology of BF2II@Lipo measured by TEM, insert was a magnification manifesting an intact lipid bilayer of 8.11 nm thickness. Scale bar, I00 nm. (F) Stability of BF21I@Lipo stored at $4{ }^{\circ} \mathrm{C}$ for one month. (G) The CD spectra of BF2II@Lipo and the physical mixture (BF2II+Lipo), where the solid lines were in corresponding to the left $y$-axis and dashed line to right y-axis. (H) The DSC graph of BF2II, Lipo, BF2II@ @Lipo and the physical mixture. (I) Percentage of cumulative release of BF2II (aq) and BF2II@Lipo at $37^{\circ} \mathrm{C}$ in PBS with different pHs. All data were presented as mean \pm SD, n=3.

was $24 \%$, the prepared liposomes had the longest half-life. The optimized ultrasonic conditions were $30 \%$ power $(200$ W) and 10 min with a $1 \mathrm{~s}$ interval for homogenization of liposomes; the incubation conditions of BF211(aq) and Lipo were $20 \mathrm{~h}$ with stirring at $50^{\circ} \mathrm{C}$. Specific optimized formulations and process parameters are shown in Figure 2B. The final concentration of BF211 in BF211@Lipo was $1.2 \mathrm{mg} / \mathrm{mL}$.

\section{Characterization of BF2 I I @Lipo}

The hydrodynamic diameter of BF211@Lipo measured by DLS was $(164.6 \pm 10.3) \mathrm{nm}$, with PDI restricted to a narrow distribution of $0.185 \pm 0.017$ (Figure 2C), and the zeta potential was $(-32.25 \pm 2.39) \mathrm{mV}$ (Figure 2D). The TEM images (Figure 2E) revealed that the morphology of the liposomes was near-spherical, and the mean particle size was approximately $160 \mathrm{~nm}$. A uniform and intact lipid bilayer with a thickness of $8.11 \mathrm{~nm}$ was observed on the surface of liposomes in accordance with the previous reports. ${ }^{27}$ The EE\% of BF211@Lipo was $(93.24 \pm 2.15) \%$ and the BF211@Lipo remained stable at $4^{\circ} \mathrm{C}$ for at least one month (Figure 2F). As shown in Figure S1A, after $12 \mathrm{~h}$ incubation with plasma, the size of liposomes increased by approximately $8 \mathrm{~nm}$, mainly due to the adsorption of the plasma protein corona. ${ }^{28}$ The size remained basically constant within $48 \mathrm{~h}$ with a slight $6 \%$ decrease in $\mathrm{EE} \%$, indicating satisfactory stability of BF211@Lipo in plasma. The particle size of liposomes also remained unchanged in tumor homogenates with only a certain increase in PDI (Figure S1B), satisfying the required therapeutic concentration and stability of the products.

TEM images of liposomal BF211 did not exhibit an oliveshaped morphology similar to liposomal Dox ${ }^{29}$ which probably depended on the structure of the payload itself. Dox, an anthraquinone compound, easily forms $\pi-\pi$ packing between 
planar conjugated systems, causing axial stretching of aggregates and deformation of liposomes. ${ }^{30}$ However, BF211 is a steroid compound and lacks a conjugated structure, ${ }^{4}$ which could account for the difference in morphology with liposomal Dox. As shown in Figure 2G, loading of BF211 into ammonium sulfate-containing liposomes dramatically changed the CD spectrum compared to the physical mixture of BF211 and empty liposomes. Even if BF211 was loaded into liposomes at a ten times lower concentration, the $\mathrm{CD}$ signal was nearly the same. The changes in CD spectra demonstrated that new species occurred inside the liposomes at a low concentration of BF211. ${ }^{29}$ The DSC thermograms of BF211@Lipo and the physical mixture are shown in Figure $2 \mathrm{H}$. The endothermic peak at $210-212^{\circ} \mathrm{C}$ was attributed to melting of BF211. This peak was not present for BF211@Lipo, suggesting that the incorporation of liposomes may indeed have altered the crystallinity of BF211. ${ }^{31}$

\section{In vitro Drug Release of BF2I I@Lipo}

The release profile of BF211@Lipo was evaluated with the dialysis method at $37^{\circ} \mathrm{C}$ under various $\mathrm{pH}$ values of 5.0, 6.5, and 7.4 to stimulate the endosomal or lysosomal compartment, inflammatory or tumor microenvironment, and normal physiological environment, respectively. ${ }^{32}$ As illustrated in Figure 2I, the cumulative release of BF211(aq) exceeded $75 \%$ within the first $2 \mathrm{~h}$ at all three different $\mathrm{pH}$ values, while the BF211@Lipo only released less than 10\% at pH 7.4 or $\mathrm{pH} 6.5$, which suggested that liposomes significantly inhibited the discharge of BF211. Moreover, the increased release of BF211@Lipo at pH 5.0 was mainly attributed to the protonation of BF211 with a higher dissolution rate in the acidic environment. ${ }^{33}$ In fact, even at $\mathrm{pH} 5$, the release of BF211@Lipo after 24 h was only approximately 35\%, while it was almost stagnant at $\mathrm{pH} 7.4$ or $\mathrm{pH} 6.5$. Notably, cumulative data have confirmed that robust drug retention in liposomes is a key factor in determining good in vivo performance and efficacy. ${ }^{11}$ A fast drug release upon the large dilution that occurs in the in vitro test often forebodes a similar or worse efficacy compared to free drugs. ${ }^{34}$ Therefore, the in vitro sustained release profile of BF211@Lipo is beneficial to drug stability and safety in circulation, which is exactly what we expected.

\section{Cardiotoxicity and Acute Toxicity Assessment of BF2II@Lipo}

Accumulating data indicated at high dosage BF can induce myocardial cell arrest within a few seconds after administration by altering intracellular calcium storage in cardiomyocytes. Another study showed that the addition of BF to guinea pig papillary muscle $(0.4 \mu \mathrm{M})$ can lead to arrhythmias. $^{35}$ To evaluate the cardiotoxicity of BF211@Lipo, the surface ECG was recorded on guinea pigs (Figure 3A). As shown in Figure 3B, subtle ECG changes were observed in the BF211(aq) group, mainly manifested in flattening and inversion of the $\mathrm{T}$ wave and depression of the ST segment. As the dose increased, the PR interval was prolonged, and deaths occurred at 24 $\mathrm{h}$ post injection of $6 \mathrm{mg} / \mathrm{kg}$ (data not listed) or less than $1 \mathrm{~h}$ after the injection of $9 \mathrm{mg} / \mathrm{kg}$, which was due to ventricular fibrillation resistant to electrical cardioversion or systolic arrest. ${ }^{36}$ These results suggested that the cardiotoxicity of BF211 is an acute and dose-dependent symptom, so lowering the initial concentration exposed to the heart is beneficial to reduce the risk of cardiac damage and death. ${ }^{37}$ In contrast, there was nearly no abnormality in the ECG profile of BF211@Lipo except for the slightly increased heart rate (Figure 3C). No deaths were observed at dosages up to $9 \mathrm{mg} / \mathrm{kg}$. As shown in Figure 3C, for $\mathrm{BF} 211(\mathrm{aq})$, the heart rate at $6 \mathrm{mg} / \mathrm{kg}$ was faster than those at $4 \mathrm{mg} / \mathrm{kg}(P=0.0357)$ and $3 \mathrm{mg} / \mathrm{kg}(P=0.0129)$, and the data dispersion, which represented as relative standard deviation (RSD), showed a significant dose-dependent increase (RSDs of control, $3 \mathrm{mg} / \mathrm{kg}, 4 \mathrm{mg} / \mathrm{kg}$ and $6 \mathrm{mg} /$ $\mathrm{kg}$ group were $0.62,2.66,5.54$ and 8.66, respectively). This result was probably attributed to the irregular heartbeat at the high dose of BF211. In contrast, for liposomal BF211, the heart rate was slightly increased from $3 \mathrm{mg} / \mathrm{kg}$ to $4 \mathrm{mg} / \mathrm{kg}$, probably resulting from trace amounts of free drug, while it remained constant in response to the increased dose of BF211 from $4 \mathrm{mg} / \mathrm{kg}$ to $9 \mathrm{mg} / \mathrm{kg}$ $(P>0.05)$. The disappearance of the dose dependence implied that liposomes changed the output kinetics of BF211. These results suggested that BF211@Lipo remarkably alleviated cardiotoxicity and had better tolerance than BF211(aq).

Single-dose acute toxicity testing showed that the $\mathrm{LD}_{50}$ of BF211@Lipo (95\% confidence) was 3-fold higher than that of BF211(aq) (Table S5), revealing that the liposomal formulation had a much lower toxicity in mice and extended the therapeutic window. We also investigated the biochemical parameters in mice on day 15 after repeated administration. $\mathrm{CK}$ and $\mathrm{LDH}$ are cardio-specific markers for ischemic cardiac injury and myocardial cell injury. $^{38}$ Animals exposed to BF211(aq) showed a significant increase in the levels of $\mathrm{CK}$ and $\mathrm{LDH}$ 
A

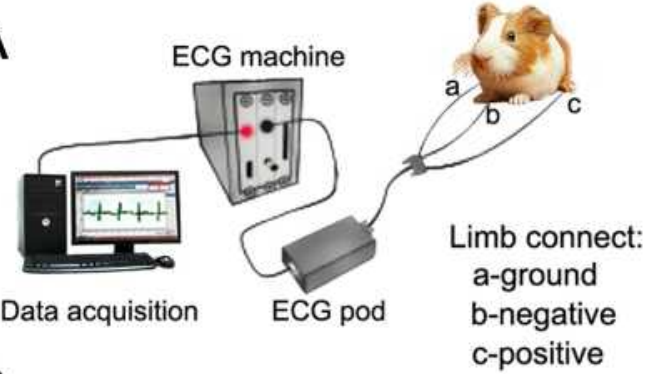

C

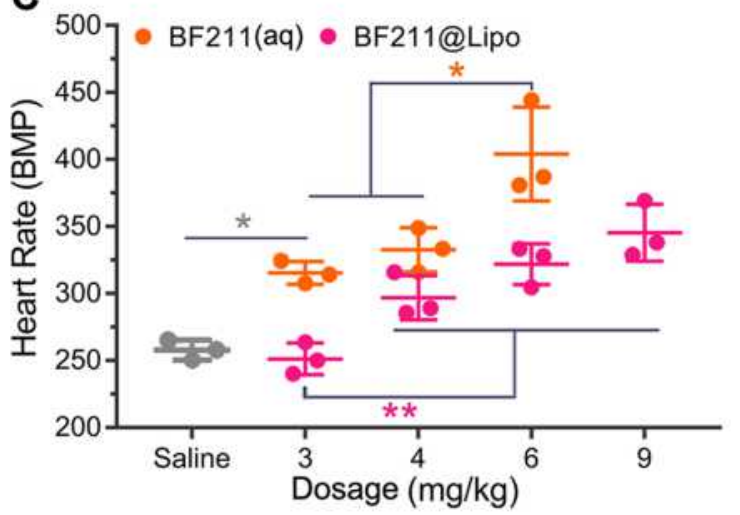

D

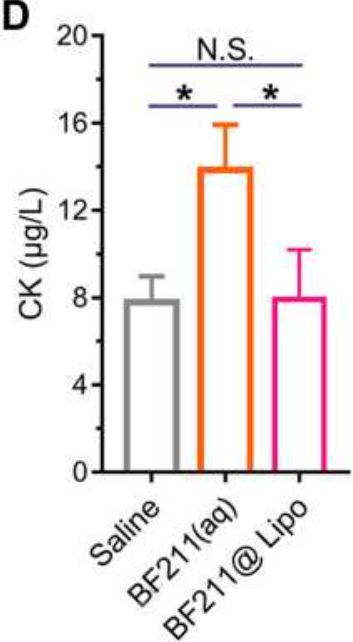

E

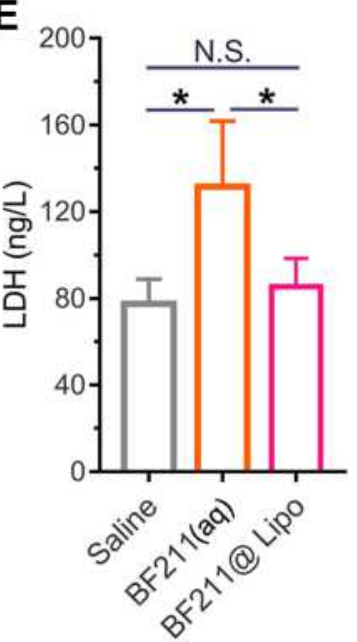

B

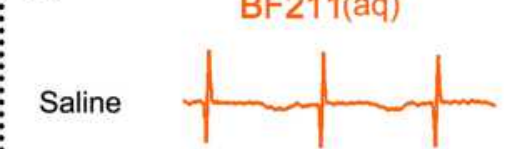
BF211@Lipo

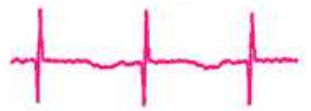

$3 \mathrm{mg} / \mathrm{kg}$

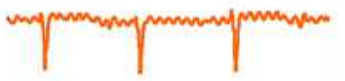

\section{$4 \mathrm{mg} / \mathrm{kg}$}

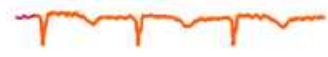

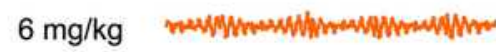

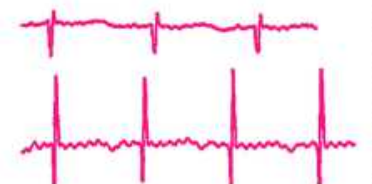

$9 \mathrm{mg} / \mathrm{kg}$ The animals were dead.

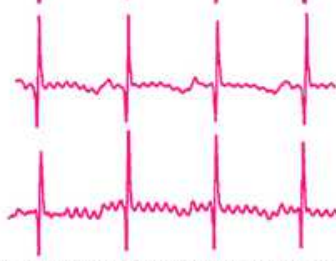

$\mathbf{F}$

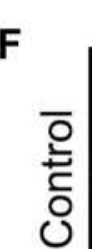

$\mathrm{AO}$ EB

Merge

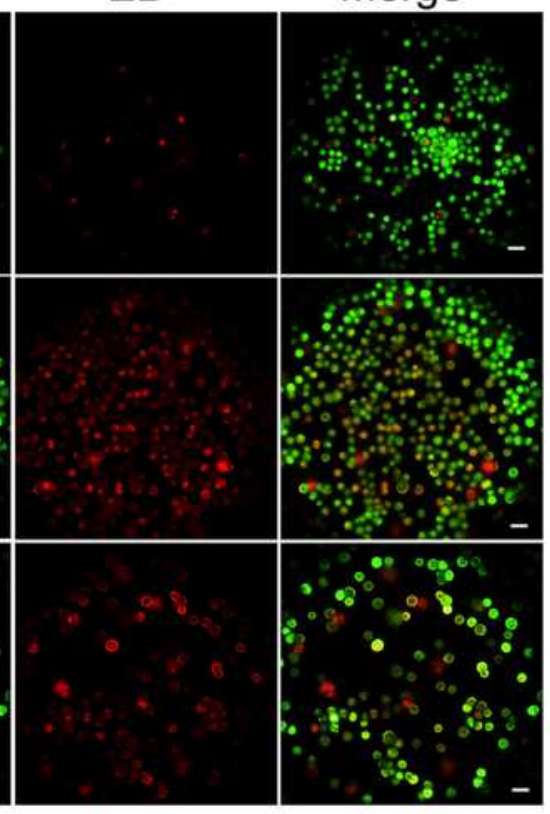

Figure 3 Cardiotoxicity and biosafety assessment of BF2II@Lipo on pigmented guinea pigs and mice. Schematic of electrocardiograms (ECGs) test (A), ECGs graphs (B) and heart rate (BPM) (C) recorded in guinea pigs I h post intraperitoneal injection. CK (D) and LDH (E) levels of mice on day I5 after intravenous (iv) injection of BF2I I (aq) or BF2II @Lipo. Mice dosed at $7 \mathrm{mg} / \mathrm{kg}$ with respect to BF2I I content of each formulation every other day for 2 weeks. (F) Dual AO/EB fluorescent staining of H9C2 cells after BF2I I (aq) and BF2II @Lipo treatments $(20 \mu \mathrm{M}, 24 \mathrm{~h})$. Live cells, live apoptotic cells and dead cells were recognized by the colors green, yellow and red. Scale bars: 75 $\mu \mathrm{m}$. All data were presented as mean $\pm S D, n=3, * p<0.05$, ** $p<0.01$.

(Figure 3D and E), probably implying increased myocardial cell necrosis. ${ }^{39}$ In contrast, this phenomenon was not observed in mice treated with BF211@Lipo. Furthermore, $\mathrm{AO} / \mathrm{EB}$ staining in H9C2 demonstrated that BF211@Lipo presented less potency to induce healthy cells death than BF211(aq) (Figure 3F). Taken together, liposomal encapsulation of BF211 can effectively diminish the toxic side effects, especially cardiotoxicity, which was probably ascribed to the reduced nonspecific distribution and decreased exposure of the heart to drugs.

\section{In vivo PK and Biodistribution of BF2II@Lipo}

The PK study of BF211@Lipo and BF211(aq) was performed on SD rats. The drug concentration of BF211(aq) in plasma almost reached to the limit of detection at 12 
h post injection, whereas BF211@Lipo remained quantifiable for at least $48 \mathrm{~h}$ (Figure S2). The decreased drug concentration of $\mathrm{BF} 211(\mathrm{aq})$ in the blood resulted in a rapid distribution to the peripheral compartment and a 23-fold larger volume of distribution (V) than that of BF211@Lipo (Table 1), which might cause unexpected side effects. ${ }^{40}$ In contrast, BF211@Lipo remained a longer half-life (11.56 $\mathrm{h}$ vs $4.59 \mathrm{~h}$ for free BF211), yielding areas under the concentration time curve (AUC) 57-fold higher than those of the BF211(aq) group $(p<0.001)$. The drug clearance (CL) of BF211@Lipo was reduced 50-fold compared to that of BF211(aq) $(p<0.001)$, which verified that the BF211@Lipo had favorable stability in blood circulation.

Biodistribution of BF211@Lipo in HepG2 xenografts was monitored in comparison to BF211(aq) by nearinfrared (NIR) fluorescence imaging, in which DiR signals can be keenly detected. As presented in Figure 4A, the fluorescence signals were quickly eliminated in free DiR group, whereas the signals in mice receiving DiR-Lipo was maintained at a high level for up to $48 \mathrm{~h}$ postinjection. Notably, DiR-Lipo showed a significantly increased tumor accumulation. At $24 \mathrm{~h}$ and $48 \mathrm{~h}$ postinjection, the tumors were excised for imaging. The results demonstrated that the fluorescence signals in the tumors from the mice treated with DiR-Lipo were approximately 4.5 times stronger than those from the mice receiving free DiR group at $48 \mathrm{~h}$ post-injection, respectively (Figure 4B and $\mathrm{C}$ ). Based on the comprehensive analysis of tissue distribution and cardiac safety evaluation results, the mechanism of reducing side effects of BF211@Lipo may be mainly attributed to its modification of drug distribution behavior in vivo, improvement of tumor targeting efficiency, and delay and reduced the release of drugs in

Table I Calculated PK Parameters of BF2II Following Single Intravenous Injection of BF2II(Aq) and BF2II@Lipo in Rats ${ }^{\mathrm{a}}$

\begin{tabular}{|l|c|c|}
\hline Parameter & BF2 I I (aq) & BF2 I I @Lipo \\
\hline AUC $_{0 \text {-inf }}(\mathrm{h} \cdot \mu \mathrm{g} / \mathrm{mL})$ & $\mathrm{I} 5.2 \mathrm{I} \pm 2.83$ & $838.23 \pm \mathrm{I} 48.77^{* * * *}$ \\
\hline $\mathrm{T}_{\mathrm{I} / 2}(\mathrm{~h})$ & $4.59 \pm 0.62$ & $\mathrm{II} .56 \pm \mathrm{I} .24^{* *}$ \\
\hline $\mathrm{V}(\mathrm{L} / \mathrm{kg})$ & $1.37 \pm 0.45$ & $0.06 \pm 0.01 * * *$ \\
\hline $\mathrm{CL}(\mathrm{mL} / \mathrm{h} / \mathrm{kg})$ & $200.00 \pm 40.00$ & $4.00 \pm 1.00^{* * *}$ \\
\hline $\mathrm{C}_{\max }(\mu \mathrm{g} / \mathrm{L})$ & $5224.20 \pm 928.97$ & $8 \mathrm{I}, 040.80 \pm 4 \mathrm{II} 6.24 * * *$ \\
\hline
\end{tabular}

Notes: ${ }^{\text {a} A n i m a l s ~ d o s e d ~ a t ~} 5 \mathrm{mg} / \mathrm{kg}$ with respect to BF2II content of each formulation. All data were presented as mean $\pm S D, n=5, * * p<0.01$, $* * * p<0.001$.

Abbreviations: $\mathrm{CL}$, clearance; $\mathrm{V}$, volume of distribution; $\mathrm{T}_{1 / 2}$, half-life; $A \cup C$, area under the plasma concentration versus time curve. normal organs, leading to the effective suppression of the acute toxicity of cardiac glycosides.

\section{Antitumor Efficacy of BF2I I@Lipo}

To evaluate the antitumor efficacy of BF211@Lipo, xenograft mice bearing HepG2 tumors were treated intravenously with saline, BF211@Lipo or BF211(aq) every other day and monitored for 3 weeks. Dox@Lipo was used as a commercial control (Figure 5A). The results showed visible tumor regression in all drug-treated groups compared with the saline group ( $p<0.001)$ (Figure 5B-D). The tumor inhibition of BF211@Lipo showed a certain dose-dependent characteristics. At a low dose of $2 \mathrm{mg} / \mathrm{kg}$, both BF211@Lipo and BF211(aq) showed a favorable tumor inhibition effect, which was significantly better than that of Dox@Lipo ( $p<0.001)$ with tumor volume and weight as evaluation criteria.BF211@Lipo showed more potent antitumor efficiency than free BF211 $(p<$ 0.05) with a $94.01 \%$ vs $84.79 \%$ decrease in tumor mass, which was probably attributed to the longer blood circulation time and the better tumor tendency of BF211@Lipo in virtue of the EPR effect. ${ }^{41}$ When the administration dose reached 4 or 8 mg/kg, BF211@Lipo exhibited a dominant effect on tumor inhibition, almost eliminating the tumor completely in a week. In vitro cytotoxicity results showed that BF211(aq) had stronger antitumor cell proliferation capacity than BF211@Lipo (Figure S3A). This may be attributed to the inefficient release of liposomes decreasing the antitumor performance of BF211@Lipo. ${ }^{42}$ Additionally, the uptake of BF211@Lipo was evaluated on normal Raw 264.7 macrophages and HepG2 tumor cells (Figure S3B and $\mathrm{C}$ ). The results indicated that BF211@Lipo were more ingested by tumor cells, showing a certain cell selectivity, which would be beneficial to reduce phagocytosis and toxicity to normal cells, enhancing the specific antitumor therapeutic effect. During the efficacy test, no noticeable behavioral abnormality was observed in any of the experimental mice. Even in the high-dose group (8 mg/kg) of BF211@Lipo, only slight weight loss was observed ( $p<0.05$ ) (Figure S4), and this weight loss might be caused by the complete ablation of tumors or the overloaded administration of BF211@Lipo.

Histologic images demonstrated that after receiving BF211@Lipo, massive cancer cell remission occurred in the tumor tissue (Figure 5E), with no obvious pathological abnormality in other primary organs (Figure S5). Considering the above cardiotoxicity experiments, it can be concluded that BF211@Lipo have good short-term and 
A

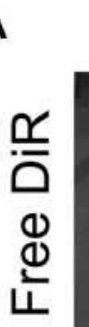

Bright

$1 \mathrm{~h}$

$2 \mathrm{~h}$

$4 \mathrm{~h}$

$8 \mathrm{~h}$

$24 \mathrm{~h}$

$48 \mathrm{~h}$
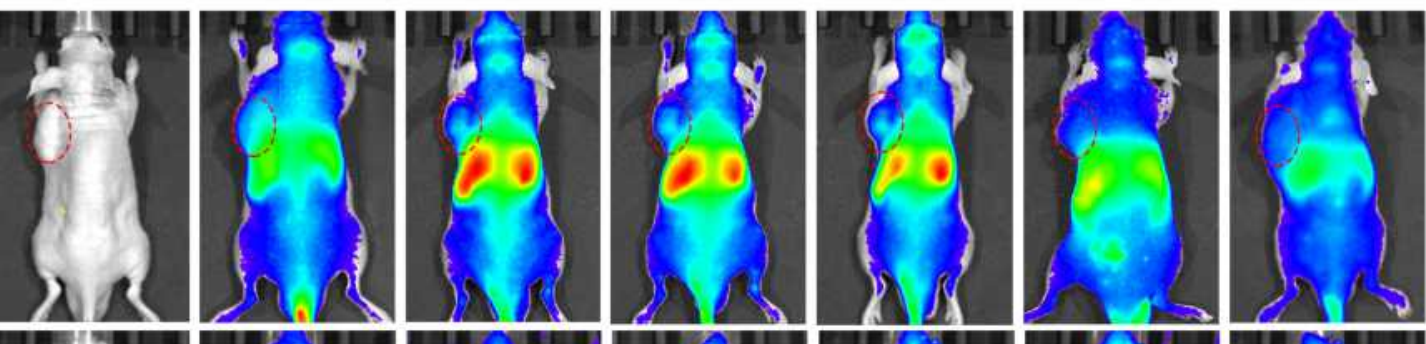

High
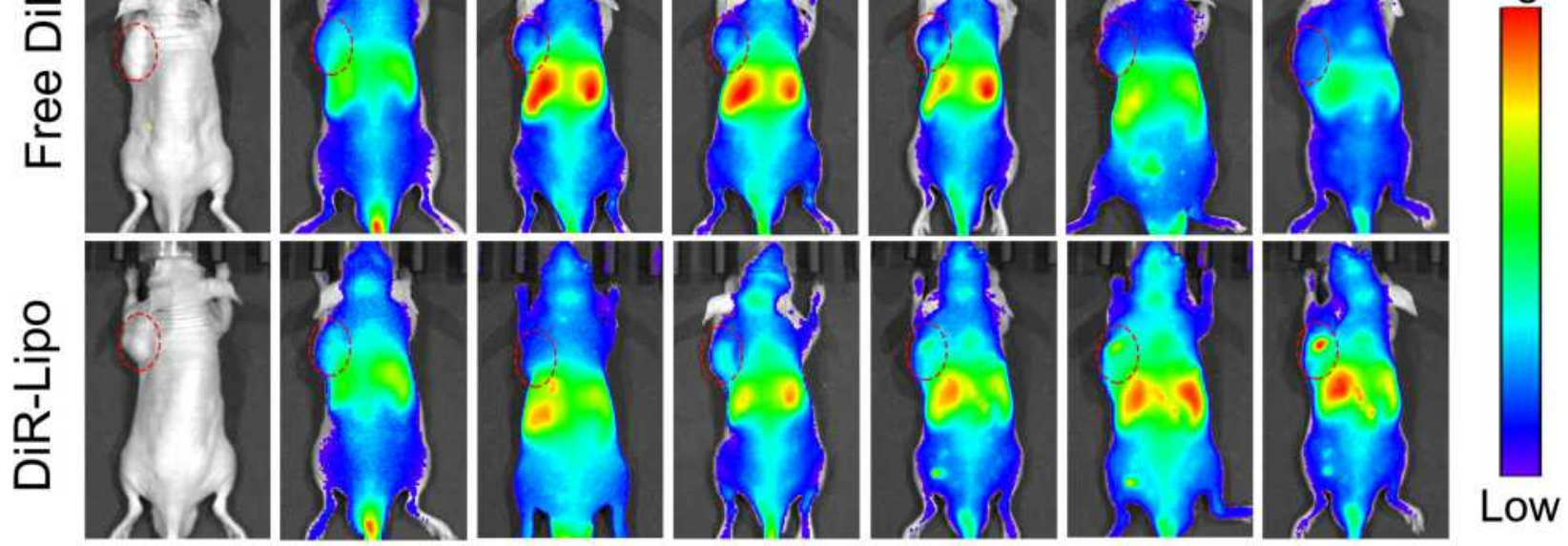

B

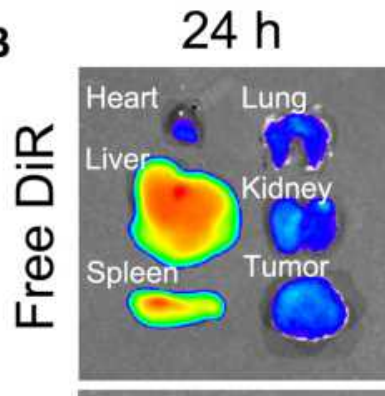

$48 \mathrm{~h}$

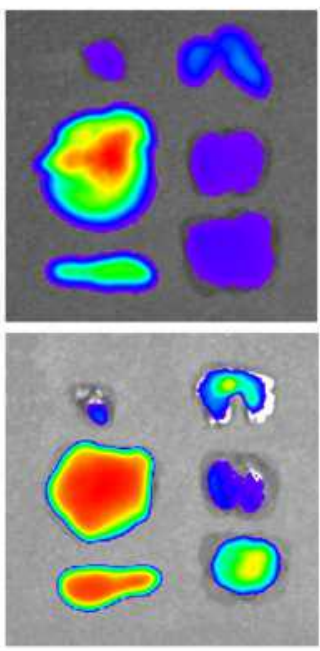

C

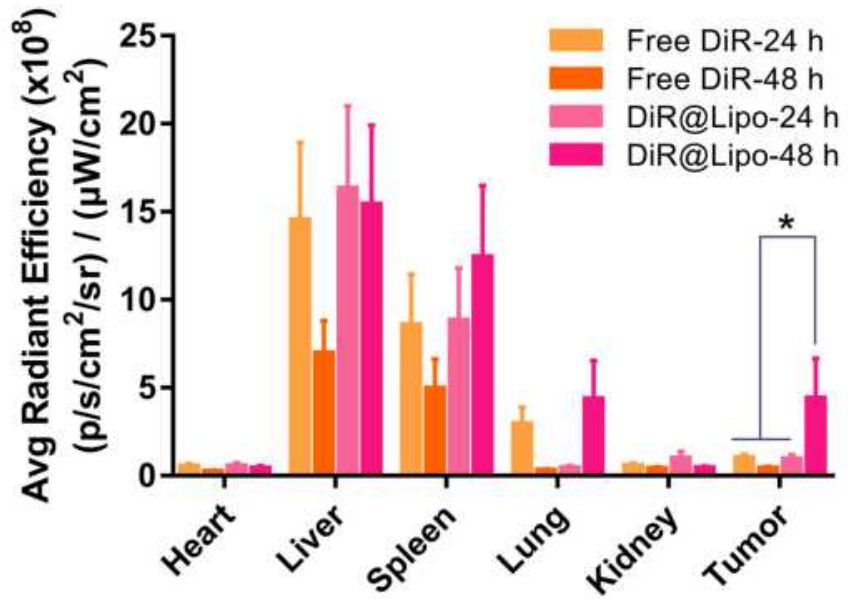

Figure 4 In vivo biodistribution of BF2I I@Lipo in a HepG2 xenograft model. (A) In vivo fluorescence imaging of tumor bearing nude mice at I, 2, 4, 8, 24 and 48 h after intravenous injection of free DiR and DiR-labelled liposomes (DiR-Lipo). Red dotted circles indicate tumor regions. (B) The dissected organs from mice at $24 \mathrm{~h}$ and 48 $\mathrm{h}$ after drug treatment were photographed. (C) Semiquantitative analysis of the fluorescence intensity from the excised organs and tumors at $24 \mathrm{~h}$ and $48 \mathrm{~h}$ post-injection. Data were represented as mean $\pm S D, n=3,{ }^{*} p<0.05$.

long-term safety. As shown by TUNEL staining (Figure 5E), tumors treated with BF211@Lipo presented the highest level of cell apoptosis, indicating that the prominent capability of tumor growth inhibition was partly due to the elevated apoptosis induced by BF211@Lipo. ${ }^{43}$ Statistical analysis of the apoptotic cells in three randomly selected tumor-section views showed that significantly more positive cells were found in the BF211@Lipo groups than in the BF211(aq) and Dox@Lipo groups $(p<0.001)$ (Figure 5F).

\section{Surfactant-Assisted Rapid-Release Strategy of BF2II@Lipo}

Surfactants are often used to increase the solubility of insoluble drugs and are often added to release media to achieve sink conditions. ${ }^{44}$ When surfactants are added to liposomes or amphiphilic micelles, they often disturb the membrane structure or change the composition of micelles, resulting in the instability of nanostructures and drug leakage. ${ }^{45,46}$ Therefore, surfactants were employed to trigger liposome release at the tumor site, improving the 
A

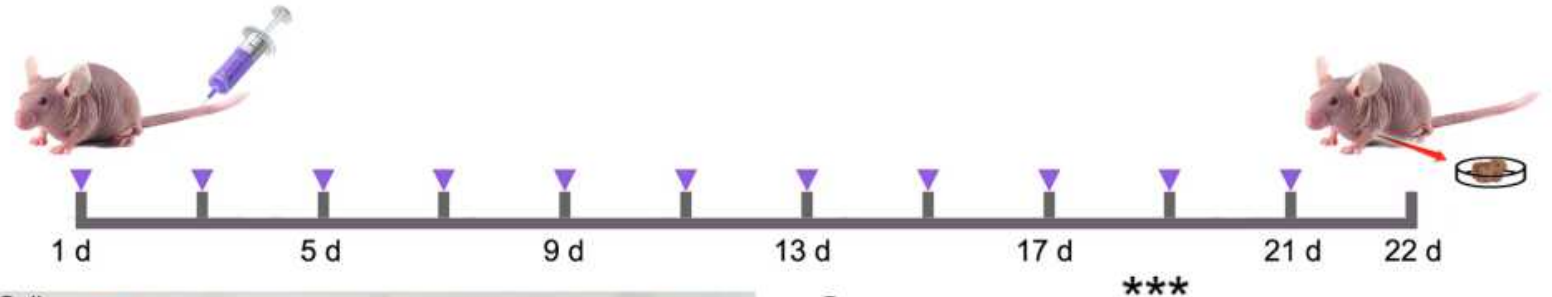

B

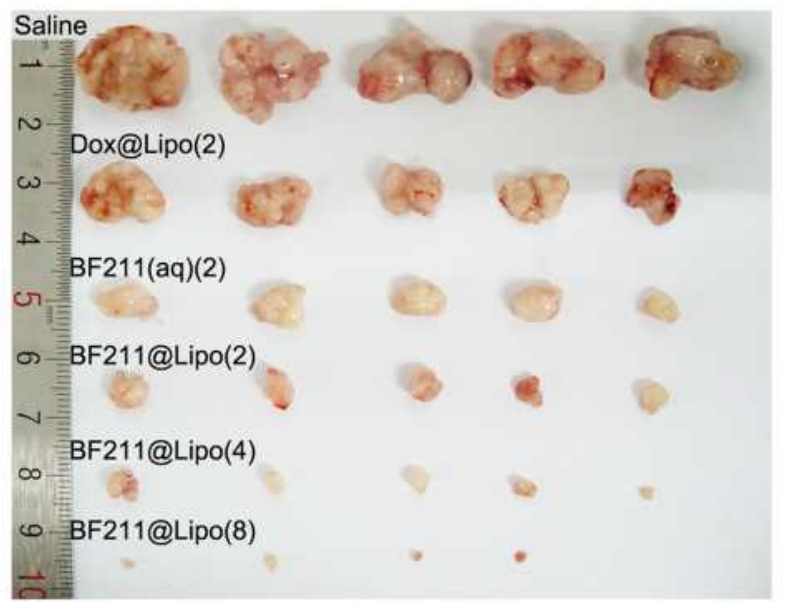

D

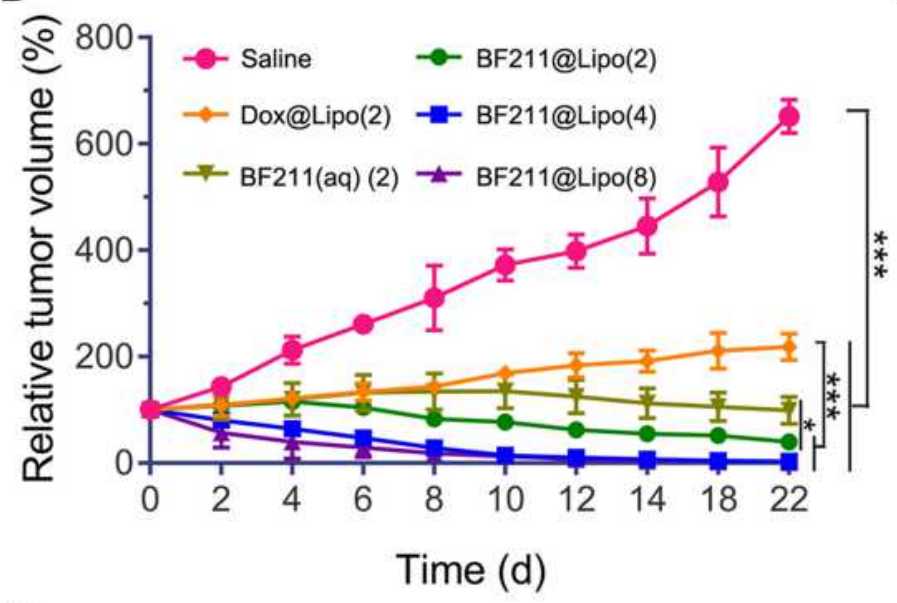

C

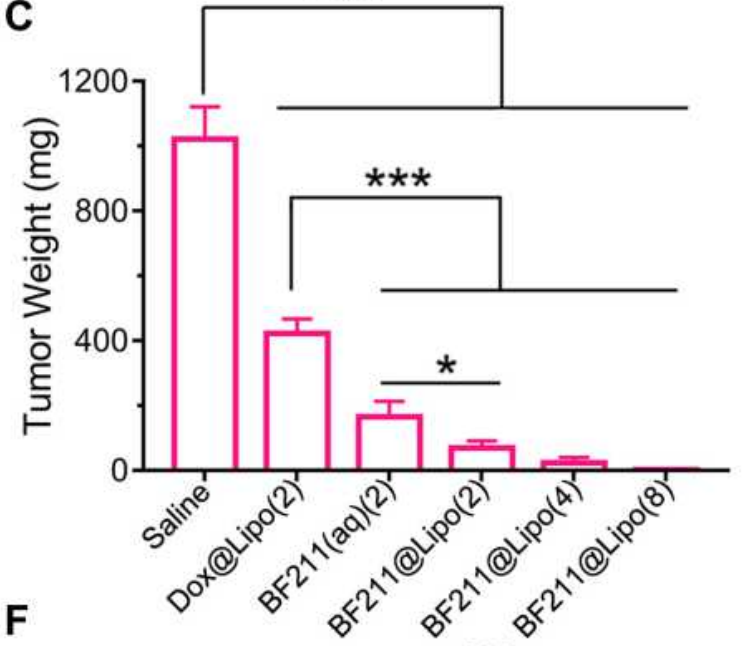

$\mathbf{F}$

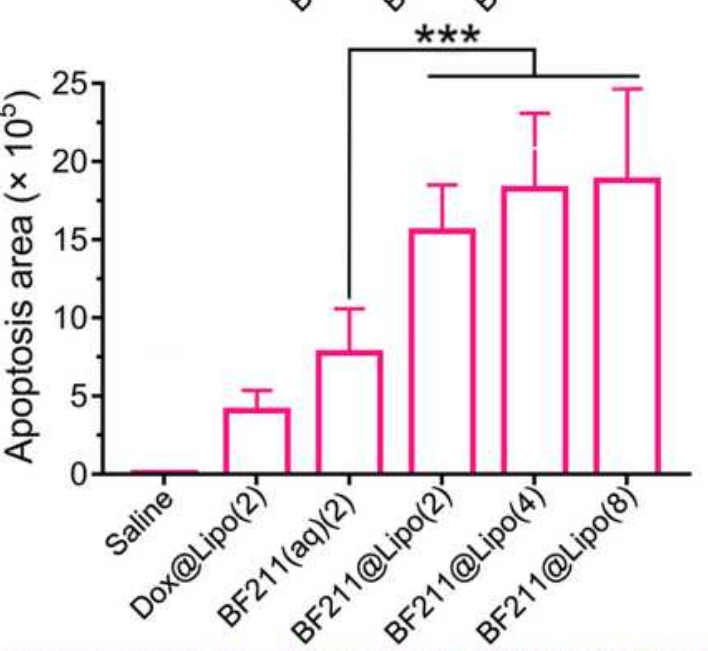

E

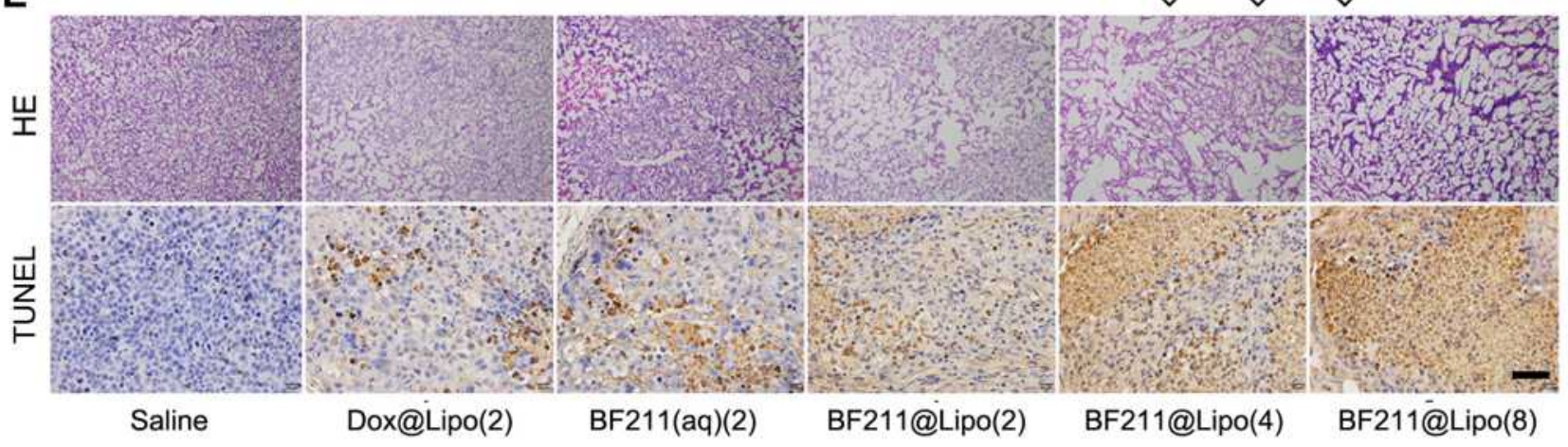

Figure 5 Antitumor efficiency of BF2II@Lipo in HepG2 murine xenograft model. (A) Schematic diagram of the dosage regimen. Violet triangle denotes the intravenous injection of saline, Dox@Lipo, BF2I I (aq) or BF2I I@Lipo. (B) Photographs of tumors harvested from mice receiving different treatments at the experimental endpoint. (C) Tumor weight in HepG2 tumor-bearing mice after 3-week repeated treatments. The numbers in brackets denote the dosage ( $\mathrm{mg} / \mathrm{kg}$ ) of different formulations. (D) Tumor growth curves over the course of the pharmacodynamics study. (E) Histological and apoptosis comparison after different treatments. Tumor sections were stained with HE for histological observation, positive TUNEL staining for apoptotic cells (brown), and DAPI for nuclei (blue). Scale bar, $50 \mu \mathrm{m}$. (F) Semiquantitative analysis of apoptosis area in the TUNEL graph with Image Pro Plus software. Data were represented as mean $\pm S D, n=5, * p<0.05, * * * p<0.001$. 
drug release of BF211@Lipo. There are few surfactants currently approved for intravenous use, of which Tween 80 and P188 are considered to be typical representatives of injectable surfactants. ${ }^{47}$ Herein, the effects of the dosage of surfactants on liposome release were investigated. As shown in Figure 6A, P188 promoted liposome release in the range of $0.005 \%$ to $1.25 \%$. In particular, when the concentration was in the range of $0.005 \%$ to $0.1 \%$, the release-promoting effect was strongest. In contrast, Tween 80 displayed a weak and unregulated release-promoting effect with no obvious optimal threshold (Figure S6). Therefore, P188 (0.05\%, w/v) was selected as a trigger to promote liposome release at the tumor site.

Subsequently, the cumulative release profile of BF211@Lipo was investigated in the presence of surfactants. As shown in Figure 6B, there was a certain increase
A

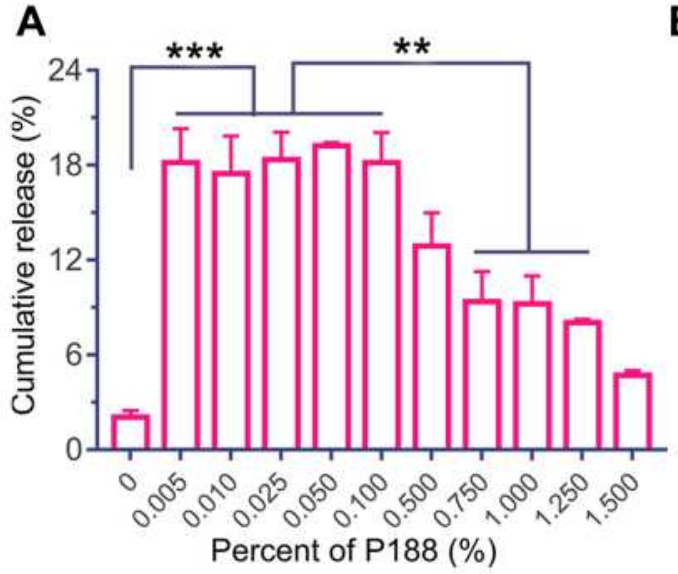

D

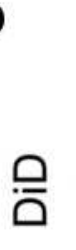

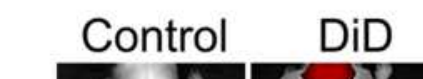

B

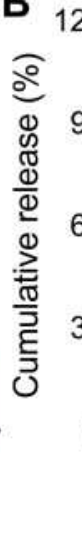

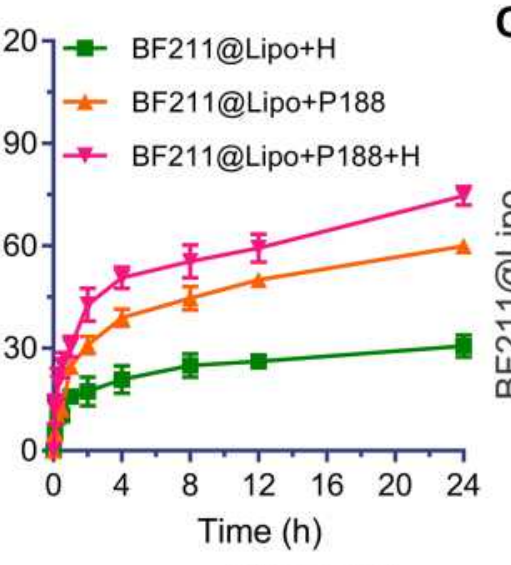

$\mathrm{DiD} / \mathrm{DiR}$
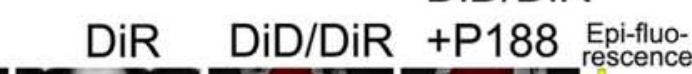

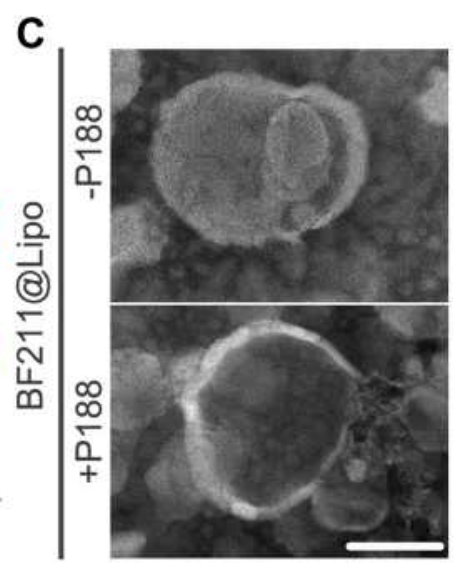

E
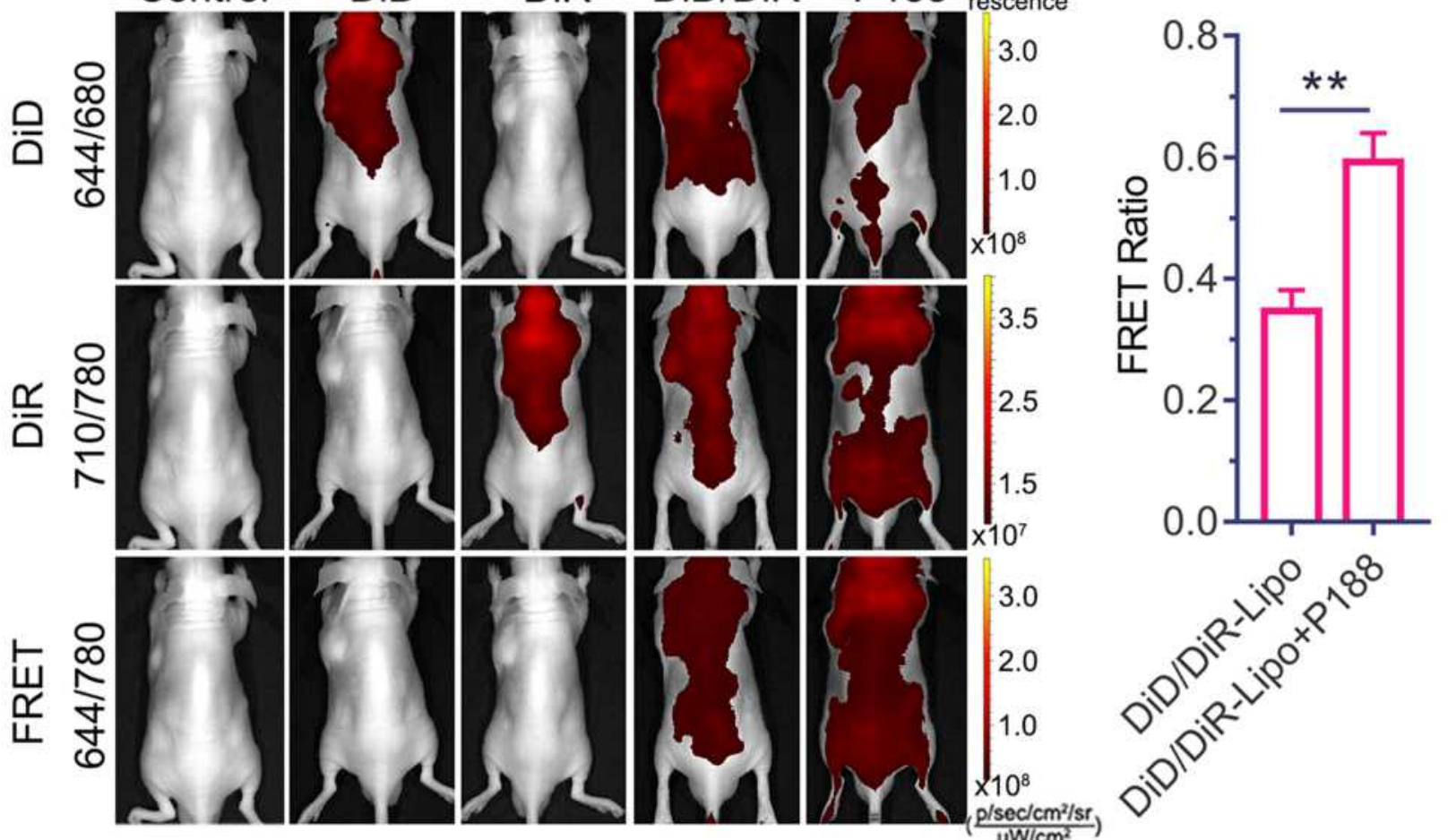

Figure 6 Scheme optimization of PI88-assisted rapid-release of BF2II@Lipo. (A) The effects of PI88 with different concentrations on the release of BF2II@Lipo after incubation at $37^{\circ} \mathrm{C}$ for $2 \mathrm{~h}$. (B) Percentage of cumulative release of BF2II@Lipo in the presence of PI88 or tumor homogenates (H) at $37{ }^{\circ} \mathrm{C}$. (C) TEM images of BF2II@Lipo and BF2II@Lipo incubated with PI88 $\left(0.05 \%\right.$, w/v) for $2 \mathrm{~h}$ at $37{ }^{\circ} \mathrm{C}$. Scale bar, $100 \mathrm{~nm}$. (D) In vivo FRET imaging of DiD and DiR release from liposomes in HepG2 tumor-bearing mice at $2 \mathrm{~h}$ post intravenous injection of PI88 $(0.05 \%$, w/v). (E) Average FRET ratios measured in the tumor regions and calculated using the automatic ROls tool. Data were represented as mean $\pm \mathrm{SD}, \mathrm{n}=3, * * p<0.0 \mathrm{I}$, *** $p<0.00 \mathrm{I}$. 

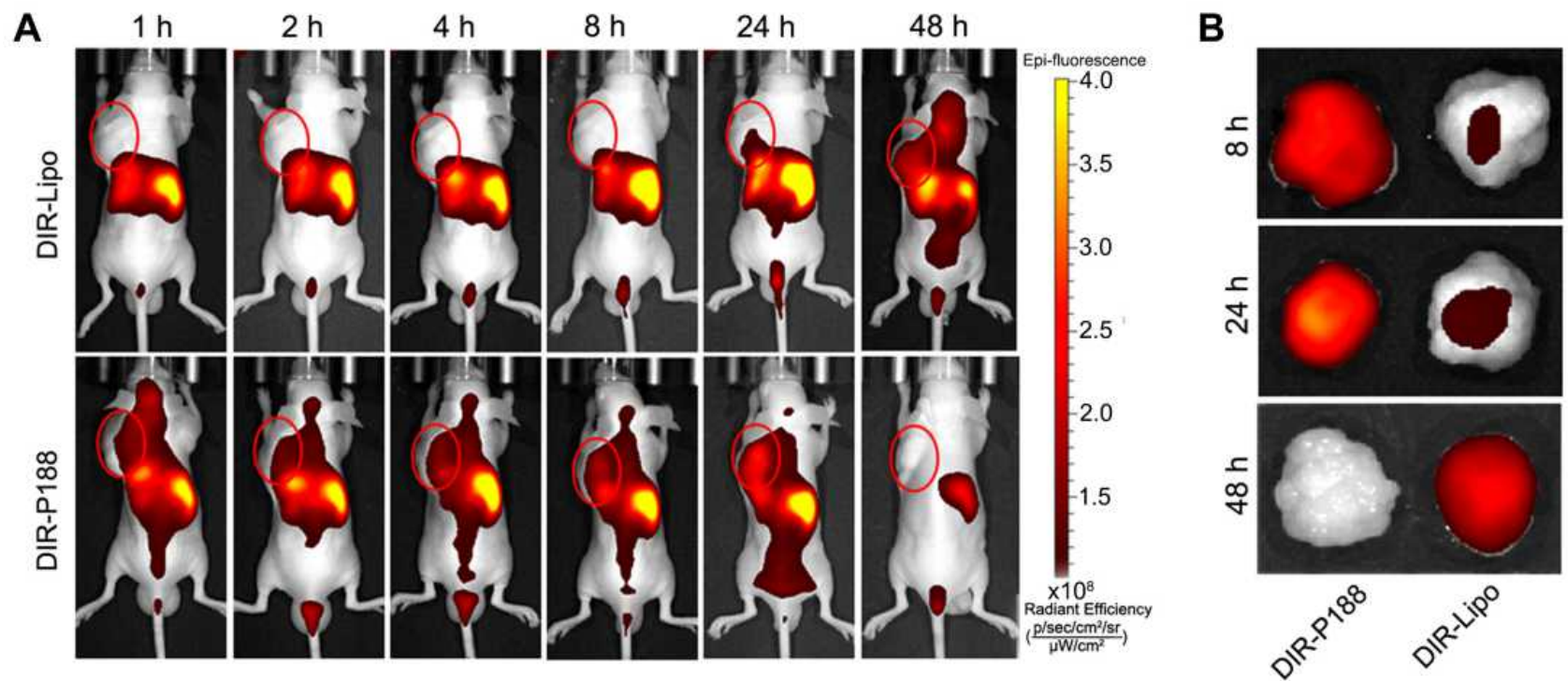

Figure 7 Monitor the accumulation of BF2II@Lipo in the tumors. (A) Biodistribution of DiR-PI88 or DiR-Lipo monitored in HepG2 tumor-bearing mice using a noninvasive near infrared imaging system. The red circles represent the tumor regions. (B) Ex vivo fluorescence images of tumors collected from mice at 8,24 and 48 $h$ post injection of DiR-PI88 or DiR-Lipo. Data were represented as mean \pm SD, $n=3$.

in the release of BF211@Lipo after incubation with tumor homogenates (30\% within $24 \mathrm{~h}$ ), which may be due to the presence of a large number of surfactant biomolecules in the tumor regions. ${ }^{48,49}$ Comparing with the results of liposomes incubated with serum (Figure S1A), it seems that liposomes are more likely to be released at tumor sites. However, the results clearly demonstrated that the release of BF211@Lipo was very limited even in the complex tumor microenvironment. However, after adding P188 to the tumor homogenates, the release of BF211@Lipo was significantly increased to nearly 75\% for $24 \mathrm{~h}$, suggesting that the release-promoting properties of $\mathrm{P} 188$ could be locally effective at the tumor sites.

The interaction mechanism of P188 and liposomes was further investigated. The results detected by DLS and flNTA showed that the addition of P188 did not change the size distribution of liposomes (Table S6 and 7). TEM results showed that after $\mathrm{P} 188$ was added to liposomes and incubated for $2 \mathrm{~h}\left(37^{\circ} \mathrm{C}\right)$, discontinuous segments appeared on the lipid bilayer, indicating the heterogeneity of the liposomal membrane ${ }^{50}$ (Figure 6C). FRET technology was employed to investigate the structural stability of liposomes. Two fluorescence dyes, DiD (donor) and DiR (acceptor), were used for FRET imaging. ${ }^{51}$ As shown in Figure S7, the fluorescence intensity of DiD/DiR-Lipo in the presence of $\mathrm{P} 188$ showed a 10-fold increase compared to that of DiD/DiR-Lipo alone; this result was probably attributed to the increased packing density of the phospholipid bilayer caused by the insertion of P188 into the lipidic membrane, ${ }^{52,53}$ leading to the enhanced FRET effect. The findings of in vivo FRET accord with the in vitro results. With the addition of P188, DiD/DiR-Lipo exhibited a significantly higher FRET ratio of $0.59 \pm 0.04$ compared to $0.35 \pm 0.04$ for DiD/DiR-Lipo alone (Figure $6 \mathrm{D}$ and E). The variations in FRET ratio indicated the changes in compatibility between probes and membranes, such as hydrophobicity and miscibility, which was closely associated with the addition of P188. Accumulating evidence indicates that amphiphilic molecules can both prevent the formation of and dissolve previously formed aggregates. ${ }^{49}$ Nonionic surfactants are able to insert into and gradually replace lipid bilayers, leading to increased membrane permeability. ${ }^{17}$ Combined with the in vitro release data, we demonstrated that P188 can accelerate drug release by disturbing the composition and structure of the lipid membrane. ${ }^{54}$

In the subsequent animal experiment, to achieve the optimal release-triggering concentration of P188 at the tumor site, $0.25 \% \mathrm{P} 188$ was chosen as a trigger on the assumption of a blood volume of $5.94 \mathrm{~mL} / 100 \mathrm{~g}$ and a weight of 20-25 g per mouse. ${ }^{55}$ The dosing interval of BF211@Lipo and P188 was optimized using in vivo imaging techniques. The results showed that the fluorescence intensity of DiR-Lipo in tumors reached a maximum at 48 $\mathrm{h}$ post intravenous injection, while DiR-P188 reached the tumors at $2 \mathrm{~h}$ post intravenous injection and remained for 
A

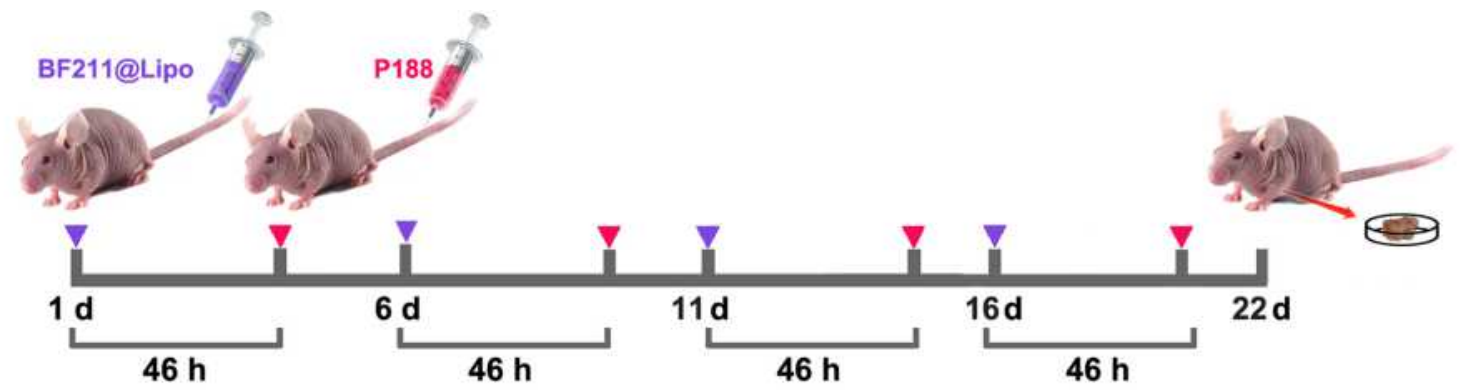

B

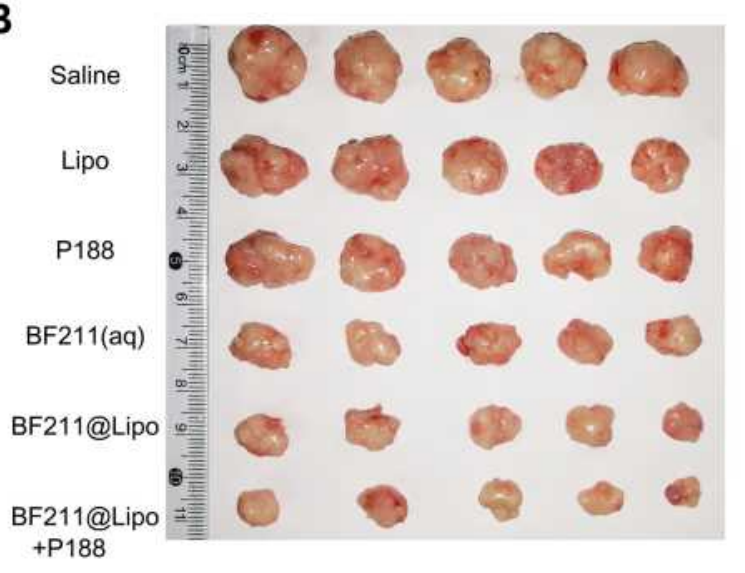

C
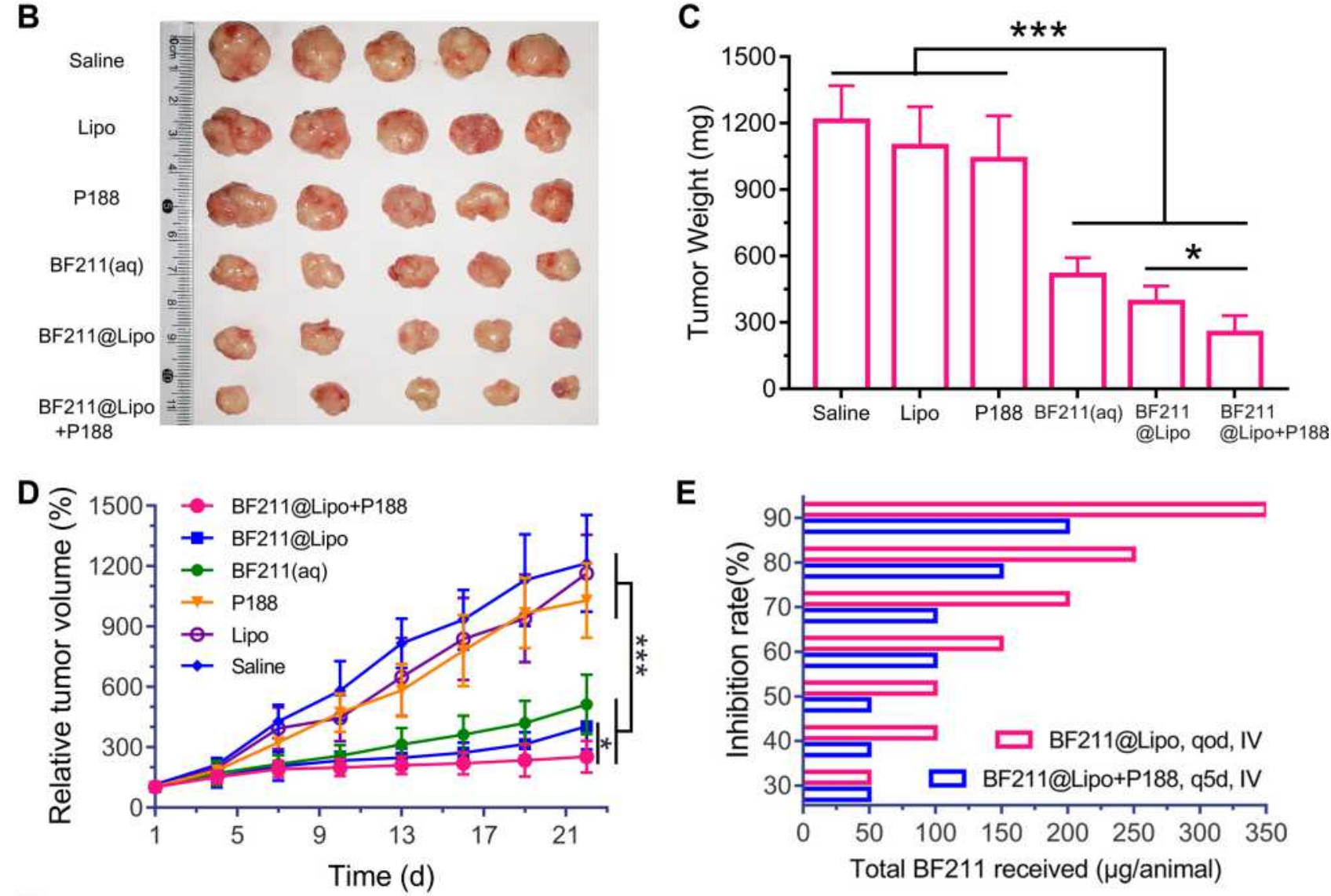

F

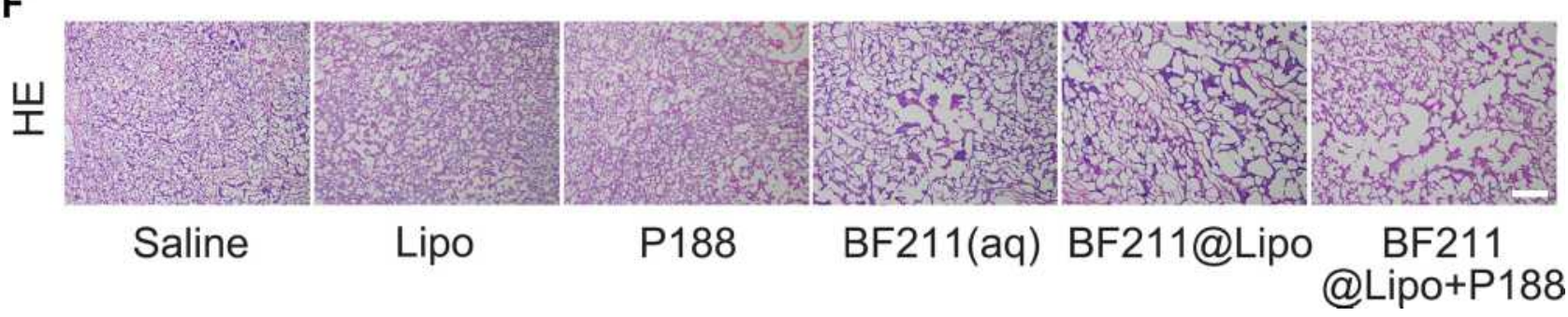

Figure 8 Antitumor efficacy of intermittent treatment schedules of BF2II@Lipo and PI88 in HepG2 murine xenograft model. (A) Schematic diagram of the dosage regimen. Violet triangle denotes the intravenous injection of BF2II@Lipo. Carmine triangle denotes the injection of $0.25 \%$ PI88 at 46 h post BF2II@Lipo administration. (B) Photographs of tumors harvested from mice at the experimental endpoint. (C) Tumor weight in HepG2 tumor-bearing mice after 3-week repeated treatments. (D) The relative tumor volume over the course of the pharmacodynamics study. (E) Tumor inhibition rate of two dosage regimens versus the total doses of BF2II administrated to mice. Qod and q5d represent every other day and every 5 days, respectively. (F) HE staining of tumor sections. Scale bar, $50 \mu \mathrm{m}$. Data were represented as mean \pm SD, $\mathrm{n}=$ $5, *_{p}<0.05, * * * p<0.001$. 
at least $24 \mathrm{~h}$ (Figure 7A and B). Finally, we developed a sequential injection of BF211@Lipo and P188 with a 46$\mathrm{h}$ interval.

\section{Antitumor Efficacy of Intermittent Treatment Schedules with BF2II@Lipo and PI88}

The in vitro cytotoxicity of BF211@Lipo combined with P188 was evaluated in HepG2 cells. As shown in Figure

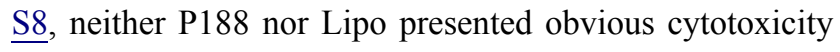
to HepG2 cells. In contrast, the addition of P188 enhanced the tumor growth inhibition capacity of BF211@Lipo compared to that of BF211@Lipo alone. These results demonstrated that P188 might trigger the release of BF211 from liposomes and increase the potent cytotoxicity of BF211.

The therapeutic regimen of intermittent dosing was determined according to preliminary screening and optimization. The whole regimen had four courses of treatment, each of which consisted of sequential injection of BF211@Lipo and P188 once with a 46-h interval (Figure $8 \mathrm{~A})$. After the treatment, the size and weight of the isolated tumors were measured. As shown in Figure 8B and $\mathrm{C}$, the combination therapy exhibited a dominant antitumor effect compared to BF211(aq) and BF211@Lipo alone. The tumor shrinkage caused by BF211@Lipo with P188 was more obvious compared with BF211(aq) ( $p<$ $0.01)$ and BF211@Lipo alone $(p<0.05)$ at the same BF211 dose of $2 \mathrm{mg} / \mathrm{kg}$ (Figure 8C and D). In contrast, Lipo and P188 had no significant tumor suppression effect similar to the saline group, demonstrating that the synergetic efficacy was mainly due to the increased drug concentration from liposomes triggered by P188 at the tumor site. The body weight of mice showed no significant change (Figure S9), indicating that there was no severe systemic toxicity caused by BF211@Lipo and P188. Furthermore, the effective BF211 dose (50\% tumor inhibition) of sequential administration (q5d, iv) was $50 \mu \mathrm{g}$ / mouse, which was only half that of BF211@Lipo alone (qod, iv) (Figure 8E), demonstrating that P188-assisted rapid release promoted the therapeutic efficiency of BF211@Lipo at the lower dose. HE staining images also supported the synergistic effect of BF211@Lipo and P188 as evidenced by the highest level of cancer cell remission in the tumor tissue (Figure 8F). In conclusion, the intermittent schedule combination therapy of BF211@Lipo and P188 resulted in synergetic antitumor efficiency, reducing the dose of chemotherapeutics and providing a comparative or even better therapeutic effect. Injectable excipients such as P188 effectively ignite "cold" liposomes locally at tumor regions to scintillate the energy against tumors similar to catalytic agents. ${ }^{56}$

\section{Conclusion}

From an active lead compound to a high-quality clinical drug, a series of careful designs and optimizations were required to meet the standards of safety, effectiveness, stability, control and compliance (the "five principles"). In this study, to solve the problem of poor in vivo targeting and severe side effects of BF211, stealth liposomes combined with an active drug loading strategy were developed under the direction of the QbD strategy. Obviously, the optimized liposomal formulation (BF211@Lipo) prolonged the blood circulation time, resulting in reduced cardiotoxicity, improved tolerance and ultimately promoted druggability. Furthermore, a P188-triggered immediate release strategy was established to enhance the release efficiency of BF211@Lipo at the tumor site. With the optimized intermittent administration schedule, BF211@Lipo exhibits both in vivo safety and antitumor activity and is obviously superior to the market available liposomal Dox. The progressive development of bufalin from a natural active lead compound to a "drug-like" candidate was achieved through efforts including structural modification, dosage form design and optimization of dosage regimen and has widened the opportunities for the research and development of high-end preparations with liposomes.

\section{Acknowledgments}

This work was supported by the National Natural Science Foundation of China (81503259), the Young Elite Scientists Sponsorship Program by CAST (2017QNRC001), Key project at central government level: The ability establishment of sustainable use for valuable Chinese medicine resources (2060302), the China and Jiangsu Postdoctoral Science Foundation (2016M591813, 1601238C), Jiangsu Six Talent Peaks Project (SWYY-057), 333 Project, QingLan Project, the Chinese National Science \& Technology Major Project "Key New Drug Creation and Manufacturing Program" (Grant 2014ZX09301-306-003), Program for Outstanding Scientific and Technological Innovation Team of Jiangsu Higher Education Institutions, the Postgraduate Research \& Practice Innovation Program of Nanjing University of 
Chinese Medicine (KYCX18_1614, KYCX20_1497, KYCX20_1498).

\section{Disclosure}

The authors report no conflicts of interest in this work.

\section{References}

1. Qi F, Li A, Inagaki Y, et al. Antitumor activity of extracts and compounds from the skin of the toad Bufo bufo gargarizans Cantor. Int Immunopharmacol. 2011;11(3):342-349. doi:10.1016/j. intimp.2010.12.007

2. Diederich M, Muller F, Cerella C. Cardiac glycosides: from molecular targets to immunogenic cell death. Biochem Pharmacol. 2017;125:1-11. doi:10.1016/j.bcp.2016.08.017

3. Lonard DM, Xu J, O'Malley BW. Bufalin is a steroid receptor coactivator inhibitor-response. Cancer Res. 2015;75(6):1157. doi:10.1158/0008-5472.CAN-14-2648

4. Lei M, Xiao Z, Ma B, et al. Synthesis and biological evaluation of bufalin-3-yl nitrogen-containing-carbamate derivatives as anticancer agents. Steroids. 2016;108:56-60. doi:10.1016/j.steroids.2016.01.011

5. Prassas I, Diamandis EP. Novel therapeutic applications of cardiac glycosides. Nat Rev Drug Discov. 2008;7(11):926-935. doi:10.1038/ nrd2682

6. Watabe M, Kawazoe N, Masuda Y, Nakajo S, Nakaya K. Bcl-2 protein inhibits bufalin-induced apoptosis through inhibition of mitogen-activated protein kinase activation in human leukemia U937 cells. Cancer Res. 1997;57(15):3097-3100

7. Han KQ, Huang G, Gu W, Su YH, Huang XQ, Ling CQ. Anti-tumor activities and apoptosis-regulated mechanisms of bufalin on the orthotopic transplantation tumor model of human hepatocellular carcinoma in nude mice. World $J$ Gastroenterol. 2007;13 (24):3374-3379. doi:10.3748/wjg.v13.i24.3374

8. Shi J, Kantoff PW, Wooster R, Farokhzad OC. Cancer nanomedicine: progress, challenges and opportunities. Nat Rev Cancer. 2017;17 (1):20-37. doi:10.1038/nrc.2016.108

9. Barenholz Y. Amphipathic weak base loading into preformed liposomes having a transmembrane ammonium ion gradient: from the bench to approved Doxil. In: Gregoriadis G, editor. Liposome Technology. 3rd. CRC Press; 2007:25-50. chap 1

10. D'Mello SR, Cruz CN, Chen ML, Kapoor M, Lee SL, Tyner KM. The evolving landscape of drug products containing nanomaterials in the United States. Nat Nanotechnol. 2017;12(6):523-529. doi:10.1038/nnano.2017.67

11. Barenholz Y. Doxil ${ }^{\mathbb{B}}$-The first FDA-approved nano-drug: lessons learned. J Control Release. 2012;160(2):117-134. doi:10.1016/j. jconrel.2012.03.020

12. Jerzy G. Active methods of drug loading into liposomes: recent strategies for stable drug entrapment and increased in vivo activity. Expert Opin Drug Deliv. 2011;8(5):565-580. doi:10.1517/ 17425247.2011.566552

13. Bolotin EM, Cohen R, Bar LK, et al. Ammonium sulfate gradients for efficient and stable remote loading of amphipathic weak bases into liposomes and ligandoliposomes. J Liposome Res. 1994;4 (1):455-479. doi:10.1016/0005-2736(93)90105-9

14. Bae YH, Park K. Targeted drug delivery to tumors: myths, reality and possibility. J Control Release. 2011;153(3):198-205. doi:10.1016/j. jconrel.2011.06.001

15. Sugahara KN, Teesalu T, Karmali PP, et al. Coadministration of a tumor-penetrating peptide enhances the efficacy of cancer drugs. Science. 2010;328(5981):1031-1035. doi:10.1126/science.1183057

16. Zamboni WC. Liposomal, nanoparticle, and conjugated formulations of anticancer agents. Clin Cancer Res. 2005;11(23):8230-8234. doi:10.1158/1078-0432.CCR-05-1895
17. Otzen DE. Biosurfactants and surfactants interacting with membranes and proteins: same but different? Biochim Biophys Acta. 2017;1859 (4):639-649. doi:10.1016/j.bbamem.2016.09.024

18. Abraham SA, Edwards K, Karlsson G, Hudon N, Mayer LD, Bally MB. An evaluation of transmembrane ion gradient-mediated encapsulation of topotecan within liposomes. $J$ Control Release. 2004;96(3):449-461. doi:10.1016/j.jconrel.2004.02.017

19. Xu X, Costa AP, Khan MA, Burgess DJ. Application of quality by design to formulation and processing of protein liposomes. Int $J \quad$ Pharm. 2012;434(1-2):349-359. doi:10.1016/j. ijpharm.2012.06.002

20. Li J, Qiao Y, Wu Z. Nanosystem trends in drug delivery using quality-by-design concept. $J$ Control Release. 2017;256:9-18. doi:10.1016/j.jconrel.2017.04.019

21. Adamson RH. The acute lethal dose 50 (LD50) of caffeine in albino rats. Regul Toxicol Pharmacol. 2016;80:274-276. doi:10.1016/j. yrtph.2016.07.011

22. Callmann CE, LeGuyader CLM, Burton ST, et al. Antitumor activity of 1,18-octadecanedioic acid-paclitaxel complexed with human serum albumin. $J$ Am Chem Soc. 2019;141(30):11765-11769. doi: $10.1021 /$ jacs. $9 \mathrm{~b} 04272$

23. Lee SW, Yun MH, Jeong SW, et al. Development of docetaxel-loaded intravenous formulation, Nanoxel-PM ${ }^{\mathrm{TM}}$ using polymer-based delivery system. J Control Release. 2011;155(2):262-271. doi:10.1016/j. jconrel.2011.06.012

24. Li P, Zhao L. Developing early formulations: practice and perspective. Int $J$ Pharm. 2007;341(1):1-19. doi:10.1016/j. ijpharm.2007.05.049

25. Rose F, Wern JE, Ingvarsson PT, et al. Engineering of a novel adjuvant based on lipid-polymer hybrid nanoparticles: a quality-bydesign approach. J Control Release. 2015;210:48-57. doi:10.1016/j. jconrel.2015.05.004

26. C`eh B, Winterhalter M, Frederik PM, Vallner JJ, Lasic DD. Stealth ${ }^{\circledR}$ liposomes: from theory to product. Adv Drug Deliv Rev. 1997;24 (2):165-177. doi:10.1016/S0169-409X(96)00456-5

27. Meng $\mathrm{H}$, Wang M, Liu $\mathrm{H}$, et al. Use of a lipid-coated mesoporous silica nanoparticle platform for synergistic gemcitabine and paclitaxel delivery to human pancreatic cancer in mice. ACS Nano. 2015;9 (4):3540-3557. doi:10.1021/acsnano.5b00510

28. Kristensen K, Engel TB, Stensballe A, Simonsen JB, Andresen TL. The hard protein corona of stealth liposomes is sparse. $J$ Control Release. 2019;307:1-15. doi:10.1016/j.jconrel.2019.05.042

29. Li X, Hirsh DJ, Cabral-Lilly D, et al. Doxorubicin physical state in solution and inside liposomes loaded via a $\mathrm{pH}$ gradient. Biochim Biophys Acta. 1998;1415(1):23-40. doi:10.1016/s0005-2736(98) 00175-8

30. Zhao Y, Chen F, Pan Y, et al. Nanodrug formed by coassembly of dual anticancer drugs to inhibit cancer cell drug resistance. ACS Appl Mater Interfaces. 2015;7(34):19295-19305. doi:10.1021/ acsami. 5 b05347

31. Franzé S, Marengo A, Stella B, Minghetti P, Arpicco S, Cilurzo F. Hyaluronan-decorated liposomes as drug delivery systems for cutaneous administration. Int $J$ Pharm. 2018;535(1):333-339. doi:10.1016/j.ijpharm.2017.11.028

32. Mo R, Sun Q, Xue J, et al. Multistage pH-responsive liposomes for mitochondrial-targeted anticancer drug delivery. Adv Mater. 2012;24 (27):3659-3665. doi:10.1002/adma.201201498

33. Russell LM, Hultz M, Searson PC. Leakage kinetics of the liposomal chemotherapeutic agent Doxil: the role of dissolution, protonation, and passive transport, and implications for mechanism of action. $J$ Control Release. 2018;269:171-176. doi:10.1016/j. jconrel.2017.11.007

34. Caddeo C, Pucci L, Gabriele M, et al. Stability, biocompatibility and antioxidant activity of PEG-modified liposomes containing resveratrol. Int $J$ Pharm. 2018;538(1):40-47. doi:10.1016/j. ijpharm.2017.12.047 
35. Cheng CS, Wang J, Chen J, et al. New therapeutic aspects of steroidal cardiac glycosides: the anticancer properties of Huachansu and its main active constituent Bufalin. Cancer Cell Int. 2019;19(1):92. doi:10.1186/s12935-019-0806-1

36. Zamiri N, Masse S, Ramadeen A, et al. Dantrolene improves survival after ventricular fibrillation by mitigating impaired calcium handling in animal models. Circulation. 2014;129(8):875-885. doi:10.1161/ CIRCULATIONAHA.113.005443

37. Richig JW, Sleeper MM. PR (PQ), QRS, QT, and other issues. In: Richig JW, Sleeper MM, editors. Electrocardiography of Laboratory Animals. Academic Press; 2014:115-116.

38. Tan LL, Lyon AR. Role of biomarkers in prediction of cardiotoxicity during cancer treatment. Curr Treat Options Cardio Med. 2018;20 (7):55. doi:10.1007/s11936-018-0641-z

39. Guo L, Luo S, Du Z, et al. Targeted delivery of celastrol to mesangial cells is effective against mesangioproliferative glomerulonephritis. Nat Commun. 2017;8(1):878. doi:10.1038/s41467-017-00834-8

40. Wada DR, Drover DR, Lemmens HJ. Determination of the distribution volume that can be used to calculate the intravenous loading dose. Clin Pharmacokinet. 1998;35(1):1-7. doi:10.2165/00003088199835010-00001

41. Maruyama K. Intracellular targeting delivery of liposomal drugs to solid tumors based on EPR effects. Adv Drug Deliv Rev. 2011;63 (3):161-169. doi:10.1016/j.addr.2010.09.003

42. Bandara SR, Molley TG, Kim H, Bharath PA, Kilian KA, Leal C. The structural fate of lipid nanoparticles in the extracellular matrix. Mater Horiz. 2020;7(1):125-134. doi:10.1039/C9MH00835G

43. Qiao H, Fang D, Zhang L, et al. Nanostructured peptidotoxins as natural pro-oxidants induced cancer cell death via amplification of oxidative stress. ACS Appl Mater Interfaces. 2018;10(5):4569-4581. doi:10.1021/acsami.7b18809

44. Williams HD, Trevaskis NL, Charman SA, et al. Strategies to address low drug solubility in discovery and development. Pharmacol Rev. 2013;65(1):315-499. doi:10.1124/pr.112.005660

45. Annesini M. Kinetics of surfactant-induced release from liposomes: a time-dependent permeability model. J Memb Sci. 2000;180 (1):121-131. doi:10.1016/s0376-7388(00)00524-X

46. Yi Z, Alakhova DY, Jong OK, Bronich TK, Kabanov AV. A simple way to enhance Doxil ${ }^{\circledR}$ therapy: drug release from liposomes at the tumor site by amphiphilic block copolymer. J Control Release. 2013;168(1):61-69. doi:10.1016/j.jconrel.2013.02.026
47. Moloughney JG, Weisleder N. Poloxamer 188 (p188) as a membrane resealing reagent in biomedical applications. Recent Pat Biotechnol. 2012;6(3):200-211. doi:10.2174/1872208311206030200

48. Tang J, Huang Y, Liu H, Zhang C, Tang D. Novel glucometer-based immunosensing strategy suitable for complex systems with signal amplification using surfactant-responsive cargo release from glucose-encapsulated liposome nanocarriers. Biosens Bioelectron. 2016;79:508-514. doi:10.1016/j.bios.2015.12.097

49. Patel A, Malinovska L, Saha S, et al. ATP as a biological hydrotrope. Science. 2017;356(6339):753-756. doi:10.1126/science.aaf6846

50. Fox C, Mulligan S, Sung J, et al. Cryogenic transmission electron microscopy of recombinant tuberculosis vaccine antigen with anionic liposomes reveals formation of flattened liposomes. Int J Nanomedicine. 2014;9:1367-1377. doi:10.2147/IJN.S56582

51. Zou P, Chen H, Paholak HJ, Sun D. Noninvasive fluorescence resonance energy transfer imaging of in vivo premature drug release from polymeric nanoparticles. Mol Pharm. 2013;10(11):4185-4194. doi: $10.1021 / \mathrm{mp} 4002393$

52. Maskarinec SA, Hannig J, Lee RC, Lee KYC. Direct observation of poloxamer 188 insertion into lipid monolayers. Biophys J. 2002;82 (3):1453-1459. doi:10.1016/S0006-3495(02)75499-4

53. Zhang W, Wang G, See E, et al. Post-insertion of poloxamer 188 strengthened liposomal membrane and reduced drug irritancy and in vivo precipitation, superior to PEGylation. $J$ Control Release. 2015;203:161-169. doi:10.1016/j.jconrel.2015.02.026

54. Zhao Y, Fay F, Hak S, et al. Augmenting drug-carrier compatibility improves tumour nanotherapy efficacy. Nat Commun. 2016;7 (1):11221. doi:10.1038/ncomms 11221

55. Vacha J. Blood volume in inbred strain BALB/c, CBA/J and C57BL/ 10 mice determined by means of $59 \mathrm{Fe}$-labelled red cells and $59 \mathrm{Fe}$ bound to transferrin. Physiol Bohemoslov. 1975;24(5):413-419.

56. Boulay A, Zumstein-Mecker S, Stephan C, et al. Antitumor efficacy of intermittent treatment schedules with the rapamycin derivative RAD001 correlates with prolonged inactivation of ribosomal protein S6 kinase 1 in peripheral blood mononuclear cells. Cancer Res. 2004;64(1):252-261. doi:10.1158/0008-5472.can-3554-2
International Journal of Nanomedicine

\section{Publish your work in this journal}

The International Journal of Nanomedicine is an international, peerreviewed journal focusing on the application of nanotechnology in diagnostics, therapeutics, and drug delivery systems throughout the biomedical field. This journal is indexed on PubMed Central, MedLine, CAS, SciSearch ${ }^{\mathbb{}}$, Current Contents ${ }^{\mathbb{R}} /$ Clinical Medicine, $^{-}$
Journal Citation Reports/Science Edition, EMBase, Scopus and the Elsevier Bibliographic databases. The manuscript management system is completely online and includes a very quick and fair peer-review system, which is all easy to use. Visit http://www.dovepress.com/ testimonials.php to read real quotes from published authors. 